LAPTH-047/15

\title{
NUT wormholes
}

\author{
Gérard Clément* \\ LAPTh, Université Savoie Mont Blanc, CNRS, 9 chemin de Bellevue, \\ BP 110, F-74941 Annecy-le-Vieux cedex, France \\ Dmitri Gal'tsov ${ }^{\dagger}$ \\ Department of Theoretical Physics, Faculty of Physics, \\ Moscow State University, 119899, Moscow, Russia \\ Mourad Guenouche $^{\ddagger}$ \\ Laboratoire de Physique Théorique, Département de Physique, \\ Faculté des Sciences Exactes, Université de Constantine 1, Algeria; \\ Department of Physics, Faculty of Sciences, \\ Hassiba Benbouali University of Chlef, Algeria
}




\begin{abstract}
We show that supercritically charged black holes with NUT provide a new setting for traversable wormholes. This does not require exotic matter, a price being the Misner string singularities. Without assuming time periodicity to make Misner strings unobservable, we show that, contrary to expectations, geodesics do not stop there. Moreover, since there is no central singularity the space-time turns out to be geodesically complete. Another unpleasant feature of spacetimes with NUTs is the presence of regions where the azimuthal angle $\varphi$ becomes timelike, signalling the appearance of closed timelike curves (CTCs). We show that among them there are no closed timelike or null geodesics, so the freely falling observers should not encounter causality violations. Considering worldlines of charged particles, we find that, although these can become closed in the vicinity of the wormhole throat for large enough charge-to-mass ratio, the non-causal orbits are still disconnected from the distant zones. Integrating the geodesic equations completely, we demonstrate the existence of timelike and null geodesics connecting two asymptotic regions of the wormhole, such that the tidal forces in the throat are reasonably small. We also discuss bounds on the NUT charge which follow from the Schwinger pair creation and ionization thresholds.

PACS numbers: 04.20.Jb, 04.50.+h, 04.65.+e
\end{abstract}

*Electronic address: gerard.clement@lapth.cnrs.fr

$\dagger$ Electronic address: galtsov@phys.msu.ru

${ }^{\ddagger}$ Electronic address: guenouche_mourad@umc.edu.dz 


\section{INTRODUCTION}

In this paper we would like to explore a novel aspect of non-vacuum space-times endowed with a Newman-Unti-Tamburino (NUT) parameter. Recall that the vacuum Taub solution was first presented as an anisotropic cosmological solution [1], and later rediscovered by Newman, Tamburino and Unti [2] as a static black hole (for more details see [3]). This latter solution has a unique event horizon of the Schwarzschild type, the corresponding internal metric being just the original Taub cosmology. Soon after Brill presented a corresponding pair for the Einstein-Maxwell system [4], its black hole face is commonly called the Reissner-NordströmNUT (RN-NUT) solution. Depending on the relative values of parameters, it can have two horizons, one degenerate horizon, or no horizon. The cosmological Brill solution corresponds to the region between two horizons in the first case. Here we investigate the third case of no horizon, which surprisingly was not discussed in the literature so far. It corresponds to a Lorentzian wormhole connecting two asymptotically locally flat spaces. Because it is free of a central singularity, there is no place for a mass or charge, so that the Brill wormhole is a realization of Wheeler's "charge without charge" and "mass without mass" [5].

An astonishing feature of this "NUT wormhole" is that it does not demand an exotic matter violating the null energy condition (NEC), or the averaged NEC (ANEC), crucial for the existence of spherical [6-8] or non spherical [9] asymptotically flat wormholes. Indeed, it is supported by a Maxwell field which is not exotic. But the price for that is the presence of a metric singularity on the polar axis known as the Misner string, which is analogous to the Dirac string of the magnetic monopole in electrodynamics. To make the string unobservable Misner suggested to impose periodicity of the time coordinate [10], which entails quantization of the energy of matter fields $[11,12]$ similar to Dirac charge quantization. This, however, leads to violation of causality throughout the space-time, so it can hardly be considered as a physically acceptable condition. In the black hole case, another problem is that analytic continuation cannot be consistently carried out through both horizons, so that the resulting spacetime is geodesically incomplete. In the wormhole case there are no horizons and analytic continuation just reduces to the extension of the semiaxis or the radial variable $r$ to the whole axis. So in the case of the Misner interpretation the wormhole RN-NUT spacetime is geodesically complete.

In an alternative interpretation of the Misner string suggested by Bonnor [13] (see also [14]), the time periodicity condition is abandoned and the polar axis is treated as a kind of topological defect with its proper matter source. We will assume this viewpoint here. Since the polar axis is now an unremovable singularity of spacetime, it was expected so far that geodesics will stop 
here. Recently we have shown [15] that it is not true for the pure Taub-NUT metric. Here we extend this proof to the Brill charged solution as well. Our proof is independent on whether one assumes the Misner or Bonnor interpretation of the space-time. In the wormhole case the space-time structure is particularly simple, and since there is no singularity other than the polar axis this means that the Brill spacetime is geodesically complete.

Also, in the Bonnor interpretation there is a region around the Misner string where closed timelike curves (CTCs) exist. Recently we have shown that in the case of the Taub-NUT spacetime these CTCs are not geodesic for a suitable choice of a parameter fixing the position of the Misner string. Thus a freely falling observer will not be confronted with causality violation. Here we extend the proof to the Brill solution too. Since we deal with charged wormholes, it is natural to consider also the worldlines of charged particles. We show that a circular worldline lying at the throat of a massless magnetically charged wormhole can be causality violating for large enough charge-to-mass ratio and a certain sign of the charge. At the same time, we show that all the wordlines extending to large distances from the throat are causal. Together with the proof of geodesic completeness, this makes us believe that NUT wormholes are free from traditional objections against solutions with NUTs.

Passing to the analysis of the physical features of the solutions, we first demonstrate the existence of timelike and null geodesics connecting two asymptotic zones. Then we investigate the tidal forces in the vicinity of the throat and show that these may be reasonably small for a large NUT charge. More restrictive bounds on this parameter are obtained by demanding that the electric field in the throat is lower than the Schwinger pair creation or the matter destruction characteristic fields. We conclude with some suggestions for further theoretical work.

\section{THE BRILL SOLUTION}

The solution of the Einstein-Maxwell system of equations found by Brill in 1964 [4] soon after the discovery of Newman, Tamburino and Unti [2] reads

$$
\begin{aligned}
d s^{2} & =-f(d t-2 n(\cos \theta+C) d \varphi)^{2}+f^{-1} d r^{2}+\left(r^{2}+n^{2}\right)\left(d \theta^{2}+\sin ^{2} \theta d \varphi^{2}\right) \\
A & =\Phi(d t-2 n(\cos \theta+C) d \varphi)
\end{aligned}
$$

with

$$
\begin{aligned}
f= & \frac{(r-m)^{2}+b^{2}}{r^{2}+n^{2}}, \quad \Phi=\frac{q r+p\left(r^{2}-n^{2}\right) / 2 n}{r^{2}+n^{2}} \\
& \left(b^{2}=q^{2}+p^{2}-m^{2}-n^{2}\right)
\end{aligned}
$$


The metric depends only on the combination $e^{2}=q^{2}+p^{2}$ of the electric $(q)$ and magnetic $(p)$ charges. For $e=0\left(b^{2}=-\left(m^{2}+n^{2}\right)\right)$ the solution reduces to the Taub-NUT solution. For $n=0$ $\left(b^{2}=e^{2}-m^{2}\right)$, it reduces to the dyonic Reissner-Nordström solution. It is particularly simple in the massless case $m=0$ for the charges satisfying $e^{2}=2 n^{2}\left(b^{2}=n^{2}\right)$, then the gravistatic potential $f(r)=1$, and the solution looks like a "pure" NUT. Finally, for $q^{2}+p^{2}=m^{2}+n^{2}$ $(b=0)$, the solution is a special case of the Israel-Wilson-Perjès (IWP) solution:

$$
\begin{aligned}
d s^{2} & =-f(d t+\overrightarrow{\mathcal{A}} \cdot d \vec{x})^{2}+f^{-1} d \vec{x}^{2}, \quad A=\Phi(d t+\overrightarrow{\mathcal{A}} . d \vec{x}), \\
f^{-1} & =1+2 \sigma \cos \alpha+\sigma^{2}, \quad \nabla \wedge \overrightarrow{\mathcal{A}}=2 \sin \alpha \nabla \sigma \\
\Phi & =\frac{f^{-1}}{2 \sin \alpha}\left[\sin \beta+2 \sigma \sin (\alpha+\beta)+\sigma^{2} \sin (2 \alpha+\beta)\right],
\end{aligned}
$$

where $\sigma(\vec{x})$ a harmonic function.

The value of the parameter $C$ introduced above can be modified by a "large" coordinate transformation

$$
t \rightarrow t+2 n\left(C-C^{\prime}\right) \varphi
$$

Such a transformation will generically lead to an intrinsically different spacetime. Thus, (2.1) actually defines a one-parameter family of spacetimes, to which we will refer collectively as "the Brill spacetime". We will keep this parameter $C$ free until section 6 .

In the case of a non-vanishing NUT charge, $n \neq 0$, the Brill solution (2.1) is not singular at $r=0$, as can be checked by computing the quadratic curvature invariants,

$$
\begin{aligned}
R^{\mu \nu} R_{\mu \nu} & =\frac{4 e^{4}}{\left(r^{2}+n^{2}\right)^{4}}, \\
R^{\mu \nu \rho \sigma} R_{\mu \nu \rho \sigma} & =\frac{8 e^{4}}{\left(r^{2}+n^{2}\right)^{4}}+\frac{48}{\left(r^{2}+n^{2}\right)^{6}}\left\{\left(m^{2}-n^{2}\right)\left[r^{6}-15 n^{2} r^{4}+15 n^{4} r^{2}-n^{6}\right]\right. \\
& -2 m r\left[\left(e^{2}-6 n^{2}\right) r^{4}-\left(10 e^{2}-20 n^{2}\right) n^{2} r^{2}+\left(5 e^{2}-6 n^{2}\right) n^{4}\right] \\
& \left.+e^{2}\left[\left(e^{2}-10 n^{2}\right) r^{4}-2\left(3 e^{2}-10 n^{2}\right) n^{2} r^{2}+\left(e^{2}-2 n^{2}\right) n^{4}\right]\right\} .
\end{aligned}
$$

The Kretschmann scalar (2.6) (where the first and second terms are respectively the Ricci square and Weyl square contributions), reduces to that of the Reissner-Nordström solution [16] for $n^{2}=0$, and to that of the Schwarzschild-NUT solution [17] for $e^{2}=0$. In view of its nonsingularity, the Brill solution can be considered as a regularization of the Reissner-Nordström solution. For $b^{2}<0$ it has, just as the RN solution, two horizons. For $b^{2}=0$, it has, just as the extreme RN solution, a double horizon. However for $b^{2}>0$, contrary to the RN solution, it is not singular, but has the (Lorentzian) wormhole topology, the coordinate $r$ varying in the whole real axis, with two asymptotic regions $r= \pm \infty$. As $r>0$ decreases, 2-spheres $r=$ constant shrink until a minimal sphere of area $4 \pi n^{2}$ (the wormhole neck) for $r=0$, and then 
expand as $r<0$ continues to decrease. Because of the absence of a point singularity, there is no source for the various (gravi-)electric and (gravi-)magnetic fluxes, and thus this solution realizes the Wheeler program [5] of mass without mass, charge without charge, etc. (for a more recent discussion see [18]. Note also that (as in the Taub-NUT case $e=0$ ), the mass parameter $m$ is not positive definite, as the reflection $r \rightarrow-r$ changes its sign and that of the electric charge $q$.

The price to pay for this regularization of the RN point singularity is the introduction of the Misner string singularity. The "Misner string" (which coincides with the Dirac string for $p \neq 0)$ consists of two disconnected infinite components, the North string piercing the North poles $\theta=0$ of all the spheres $r=$ constant $(r \in R)$ and its counterpart the South string for $\theta=\pi$, except in the special gauges $C=\mp 1$, where only one component (South or North) is singular. The Misner string singularity can be transformed away altogether if the time coordinate $t$ is periodically identified with period $8 n \pi$. However there is also a price to pay for this. First, there are closed timelike curves (CTC) everywhere in all the Brill spacetimes. Second, in the case $b^{2} \leq 0$, the Kruskal continuation cannot be consistently carried out with this periodical identification, so that the black-hole Brill spacetimes are not geodesically complete $[3,19]$, i.e. the Misner string singularity has been traded for a singular horizon.

So let us keep the usual real time axis, thereby retaining also the Misner string. In this case it has been argued [19] (in the case of the Taub-NUT black hole spacetime, but exactly the same argument could be formulated in the general Brill case) that geodesics must terminate at the Misner string singularity, just because this is a metric singularity. Actually, geodesic motion does not depend on the metric itself, but only on the connections, so a metric singularity does not necessarily imply geodesic termination. Geodesics do indeed terminate at a conical singularity (the analogy made in [19]), but not at the metric singularity $r=0$ of the plane with metric $d l^{2}=d r^{2}+r^{2} d \theta^{2}$ ! The investigation of geodesic motion in the metric (2.1), carried out in the next section, will show 1) that all geodesics which hit the Misner string cross it smoothy, so that all the Brill spacetimes with $n^{2} \neq 0$ are geodesically complete; 2) that if furthermore $b^{2}>0$ the resulting Lorentzian wormholes are traversable. 


\section{GEODESICS: ANGULAR AND TEMPORAL MOTION}

The partially integrated geodesic equations for the metric (2.1) are [20]

$$
\begin{aligned}
& f(r)(\dot{t}-2 n(\cos \theta+C) \dot{\varphi})=E, \\
& \dot{\varphi}=\frac{L_{z}(\theta)}{\left(r^{2}+n^{2}\right) \sin ^{2} \theta}, \\
& {\left[\left(r^{2}+n^{2}\right) \dot{\theta}\right]^{\cdot}=\left(r^{2}+n^{2}\right) \sin \theta \cos \theta \dot{\varphi}^{2}-2 n E \sin \theta \dot{\varphi},} \\
& f(r)^{-1}\left(\dot{r}^{2}-E^{2}\right)+\left(r^{2}+n^{2}\right)\left(\dot{\theta}^{2}+\sin ^{2} \theta \dot{\varphi}^{2}\right)=\varepsilon,
\end{aligned}
$$

where ${ }^{\cdot}=d / d \tau$, with $\tau$ an affine parameter,

$$
L_{z}(\theta)=J_{z}-2 n E \cos \theta
$$

and $E$ and $J_{z}$ are the constants of the motion associated with the cyclic variables $t$ and $\varphi$ (see Appendix A). In the following we assume without loss of generality that for timelike and null geodesics $E>0$, thereby defining the orientation of time, and $n>0$, the sign of $J_{z}$ remaining arbitrary. Eq. (3.4) is $d s^{2}=\varepsilon d \tau^{2}$, with $\varepsilon=-1,0$ or 1 for timelike, null or spacelike geodesics, respectively.

The analysis of geodesic motion in the Brill metric parallels that of a charged particle in the field of a magnetic monopole [20]. Similarly to the case of the Taub-NUT metric, the metric (2.1) admits four Killing vectors, one $\left(K_{0}\right)$ generating time translations, and three $\left(K_{i}\right)$ generating the rotation group $O(3)$. Due to this spherical symmetry, the angular components of the geodesic equations can be first integrated by

$$
\vec{L}+\vec{S}=\vec{J}
$$

where $\vec{J}$ is a constant vector, the total angular momentum, which is the sum of an orbital angular momentum $\vec{L}$ and a "spin" angular momentum $\vec{S}$. The components of $\vec{L}$ and $\vec{S}$ are given in [20], where it is shown that

$$
\vec{L}=\left(r^{2}+n^{2}\right) \hat{r} \wedge \dot{\hat{r}}, \quad \vec{S}=2 n E \hat{r}
$$

where

$$
\hat{r}=(\sin \theta \cos \varphi, \sin \theta \sin \varphi, \cos \theta)
$$

is a unit vector normal to the two-sphere.

It follows from the orthogonality of $\vec{L}$ and $\vec{S}$ that

$$
\vec{J} \cdot \hat{r}=2 n E .
$$


In the magnetic monopole case, such a first integral means that the the trajectory of the charged particle lies on the surface of a cone with axis $\vec{J}$ originating from the magnetic monopole source $r=0$. However the Taub-NUT or Brill gravitational field has no source, i.e. no apex for the "cone". Actually, the content of (3.9) is that the geodesic intersects all the two-spheres of radius $r$ on the same small circle, or parallel, $\mathcal{C}$ with polar axis $\vec{J}$. Squaring (3.6) leads to

$$
\vec{J}^{2}=\vec{L}^{2}+4 n^{2} E^{2}
$$

which can be rewritten as

$$
\left(r^{2}+n^{2}\right)^{2}\left[\dot{\theta}^{2}+\sin ^{2} \theta \dot{\varphi}^{2}\right]=l^{2},
$$

with $l^{2}=J^{2}-4 n^{2} E^{2}\left(J^{2}=\vec{J}^{2}\right)$. Insertion into (3.4) leads to the effective radial equation

$$
\dot{r}^{2}+f(r)\left[\frac{l^{2}}{r^{2}+n^{2}}-\varepsilon\right]=E^{2},
$$

which is identical to the equation for radial motion in the equatorial plane for the metric (2.1) without the term $-2 n \cos \theta d \varphi$, i.e. for $b^{2}>0$ motion in a static spherically symmetric wormhole geometry of the Ellis type [21] (the $m=0$ symmetrical Ellis wormhole [22] for $f(r)=1$ ).

\section{A. Angular motion}

Knowing (in principle) the solution to (3.12), one can insert it in (3.11), where $\dot{\varphi}$ can be eliminated by using (3.2), to yield a first-order differential equation for $\theta(\tau)$. Then the solution can be used to obtain $\varphi(\tau)$ and $t(\tau)$ by integrating (3.2) and (3.1). The Misner string singularities $\theta=0, \pi$ do not seem to play any role in the complete integration thus carried out. To check this, we follow [19] and replace the affine parameter $\tau$ by the new variable $\lambda$ defined by

$$
d \tau=\left(r^{2}+n^{2}\right) d \lambda
$$

which increases monotonously with $\tau$. Putting

$$
\xi=\cos \theta
$$

the differential equation for $\theta(\lambda)$ reads

$$
\left(\frac{d \xi}{d \lambda}\right)^{2}=-J^{2} \xi^{2}+4 n E J_{z} \xi+\left(l^{2}-J_{z}^{2}\right) .
$$

Assuming $J^{2} \neq 0\left(J^{2}=0\right.$ implies from (3.10) $E=0$ and $\left.l=0\right)$, Eq. (3.15) is solved (up to an additive constant to $\lambda$ ) by [19]

$$
\cos \theta=J^{-2}\left[2 n E J_{z}+l J_{\perp} \cos (J \lambda)\right]
$$


where

$$
J_{\perp}^{2}=J^{2}-J_{z}^{2}
$$

Because the constants of the motion occurring in (3.16) are related by the two Pythagorean decompositions of $\vec{J}^{2}$ (3.10) and (3.17), it will be useful to trade them for the two angles $\eta$ and $\psi$ defined by

$$
2 n E=J \cos \eta, \quad l=J \sin \eta, \quad J_{z}=J \cos \psi, \quad J_{\perp}=J \sin \psi,
$$

with $0 \leq \eta \leq \pi / 2$ and $0 \leq \psi \leq \pi$. Then (3.16) reads

$$
\cos \theta=\cos \psi \cos \eta+\sin \psi \sin \eta \cos (J \lambda)
$$

Eq. (3.15) has two turning points $\theta_{ \pm}$such that

$$
\cos \theta_{ \pm}=J^{-2}\left(2 n E J_{z} \pm l J_{\perp}\right)=\cos (\psi \mp \eta)
$$

These can be rewritten as

$$
\theta_{+}=\left|\begin{array}{ll}
\psi-\eta & \text { if } \psi \geq \eta \\
-\psi+\eta & \text { if } \psi \leq \eta
\end{array}, \quad \theta_{-}=\right| \begin{array}{cc}
\psi+\eta & \text { if } \psi \leq \pi-\eta \\
-\psi-\eta+2 \pi & \text { if } \psi \geq \pi-\eta
\end{array} .
$$

It follows that the trajectory crosses periodically the Misner string, $\cos \theta_{ \pm}= \pm 1$ only if

$$
\psi=\eta\left(J_{z}=2 n E\right) \quad \text { or } \quad \psi=\pi-\eta\left(J_{z}=-2 n E\right) .
$$

The only geodesics which can cross both components of the Misner string are those with $\eta=$ $\pi / 2\left(E=J_{z}=0\right)$, leading to $\dot{t}=\dot{\varphi}=0$; according to (3.12), in the stationary sector $(f(r)>0)$ these can only be spacelike geodesics. The trajectory can also stay on the Misner string component $\theta=0$ or $\pi$ if (3.22)) is satisfied with $\eta=0(2 n E=J)$.

The differential equation (3.2) for $\varphi$ can be rewritten as

$$
\frac{d \varphi}{d \lambda}=\frac{1}{2}\left[\frac{J_{z}-2 n E}{1-\cos \theta(\lambda)}+\frac{J_{z}+2 n E}{1+\cos \theta(\lambda)}\right]
$$

with $\cos \theta(\lambda)$ given by (3.16). This is solved by [19]

$$
\begin{aligned}
\varphi-\varphi_{0} & =\arctan \left[\frac{\cos \psi-\cos \eta}{1-\cos (\psi-\eta)} \tan \frac{J \lambda}{2}\right] \\
& +\arctan \left[\frac{\cos \psi+\cos \eta}{1+\cos (\psi-\eta)} \tan \frac{J \lambda}{2}\right],
\end{aligned}
$$

with $\varphi_{0}$ an integration constant. This can be simplified to

$$
\varphi-\varphi_{0}=\arctan \left[\frac{2 \sin \eta \tan (J \lambda / 2)}{\sin (\eta-\psi)-\sin (\eta+\psi) \tan ^{2}(J \lambda / 2)}\right] .
$$


For trajectories crossing the North Misner string, with $\psi=\eta\left(J_{z}=2 n E\right)$, this reduces to

$$
\varphi-\varphi_{1}=\arctan \left(\cos \eta \tan \left(\frac{J \lambda}{2}\right)\right)
$$

with $\varphi_{1}=\varphi_{0}-\operatorname{sgn}(\tan (J \lambda / 2)) \pi / 2$. A similar formula applies in the case of the South Misner string, with $\eta$ replaced by $\pi-\eta$ and $J \lambda$ replaced by $J \lambda-\pi$ (note that according to (3.16) the North Misner string is crossed for $\lambda=2 k \pi / J$, while the South Misner string is crossed for $\lambda=(2 k+1) \pi / J, k$ integer $)$. In the case e.g. of the North Misner string, this gives on account of $(3.16)$,

$$
\cos \left(\varphi-\varphi_{1}\right)=\frac{J_{z}}{J_{\perp}} \tan \left(\frac{\theta}{2}\right)
$$

consistent with $(3.9)$ (the choice $\varphi_{1}=0$ in (3.27) corresponds to the choice $\vec{J}=\left(J_{\perp}, 0, J_{z}\right)$ in (3.9)). Clearly the Misner string is completely transparent to the geodesic motion!

When the parameter $\lambda$ varies over a period, e.g. $\lambda \in[-\pi / J, \pi / J]$, the argument of the first or second arctan in (3.24) varies from $-\infty$ to $+\infty$ for $J_{z} \mp 2 n E>0$, and from $+\infty$ to $-\infty$ for $J_{z} \mp 2 n E<0$. It is identically zero for $J_{z} \mp 2 n E=0$. Accordingly, the variation of $\varphi$ over a period is

$$
\Delta \varphi=\pi\left[\operatorname{sgn}\left(J_{z}-2 n E\right)+\operatorname{sgn}\left(J_{z}+2 n E\right)\right] .
$$

This means that for $J_{z}^{2}>4 n^{2} E^{2}(|\Delta \varphi|=2 \pi)$ the parallel $\mathcal{C}$ circles the North-South polar axis, i.e. the Misner string. For $J_{z}^{2}<4 n^{2} E^{2},(|\Delta \varphi|=0) \mathcal{C}$ does not circle the Misner string. And for $J_{z}= \pm 2 n E(|\Delta \varphi|=\pi), \mathcal{C}$ goes through the North or South pole, as discussed above.

The two turning points (3.21) coincide if either $\psi=0$, or $\eta=0$ :

a) $\psi=0$ or $\pi$. The constant vector $\vec{J}$ is aligned with the $z$ axis, on which the parallel $\mathcal{C}$ is centered:

$$
\theta=\eta \text { or } \pi-\eta, \quad \varphi-\varphi_{0}= \pm J \lambda .
$$

As discussed in [20], the solution (3.16), (3.25) can always be rotated to this case, the Misner string being then rotated away from the $z$ axis.

b) $\eta=0$. The orbital angular momentum vanishes, $l=0$, and the motion is purely radial,

$$
\theta=\psi, \quad \varphi-\varphi_{0}=0
$$

This will be further discussed in section 4 .

\section{B. Temporal motion}

After transforming to the geodesic variable $\tau$ by (3.13), the solution to (3.1) can be written as the sum

$$
t(\lambda)=t_{r}(\lambda)+t_{\theta}(\lambda)
$$


where the radial and angular contributions to $t(\lambda)$ solve the equations

$$
\begin{aligned}
& \frac{d t_{r}}{d \lambda}=E \frac{r^{2}+n^{2}}{f(r)}, \\
& \frac{d t_{\theta}}{d \lambda}=\frac{2 n(\cos \theta+C)\left(J_{z}-2 n E \cos \theta\right)}{\sin ^{2} \theta} .
\end{aligned}
$$

The solution to equation (3.32) depends on the solution of the equation for radial motion (3.12). We consider here equation (3.33). This can be rewritten as

$$
\frac{d t_{\theta}}{d \lambda}=4 n^{2} E+n\left[\frac{(C+1)\left(J_{z}-2 n E\right)}{1-\cos \theta(\lambda)}+\frac{(C-1)\left(J_{z}+2 n E\right)}{1+\cos \theta(\lambda)}\right],
$$

with $\cos \theta(\lambda)$ given by (3.16). The explicit solution to equation (3.34) is [19]

$$
\begin{aligned}
t_{\theta}(\lambda) & =\bar{t}_{\theta}(\lambda)=4 n^{2} E \lambda+2 n(C+1) \arctan \left[\frac{\cos \psi-\cos \eta}{1-\cos (\psi-\eta)} \tan \frac{J \lambda}{2}\right] \\
& +2 n(C-1) \arctan \left[\frac{\cos \psi+\cos \eta}{1+\cos (\psi-\eta)} \tan \frac{J \lambda}{2}\right]
\end{aligned}
$$

in the interval $-\pi / J<\lambda<\pi / J$. The resulting variation of $t_{\theta}$ over a period $2 \pi / J$ of $\lambda$ is

$$
\Delta t_{\theta}=2 \pi n\left[\frac{4 n E}{J}+(C+1) \operatorname{sgn}\left(J_{z}-2 n E\right)+(C-1) \operatorname{sgn}\left(J_{z}+2 n E\right)\right]
$$

so that the solution for generic values of $\lambda$ is

$$
t_{\theta}(\lambda)=\bar{t}_{\theta}(\lambda)+\left[\Delta t_{\theta}-\frac{8 \pi n^{2} E}{J}\right] \mathrm{E}\left(\frac{J \lambda}{2 \pi}+\frac{1}{2}\right) .
$$

For parallels $\mathcal{C}$ which do not circle the Misner string $\left(J_{z}^{2}<4 n^{2} E^{2}\right), \Delta t_{\theta}$ is negative and independent of the value of $C, \Delta t_{\theta}=-4 \pi n(1-\cos \eta)$. In the limit when such parallels contract to

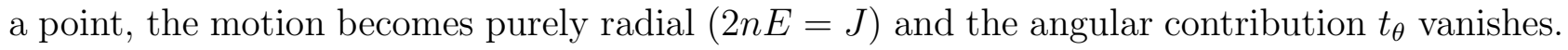

Let us recall here that, while the solution for the spatial components of the geodesic (the orbit) can be rotated to a frame in which the $z$ axis points along the total angular momentum vector $\vec{J}$, this is not the case for the time coordinate $t(\lambda)$. The correct equation for the time evolution in this rotated frame is given (for the choice $C=0$ ) in [20], Eq. (24a).

\section{GEODESICS: THE COMPLETE ORBITS}

\section{A. The radial potential}

The radial equation (3.12) can be written in the familiar form

$$
\left(\frac{d r}{d \tau}\right)^{2}+U(r)=E^{2}+\varepsilon
$$




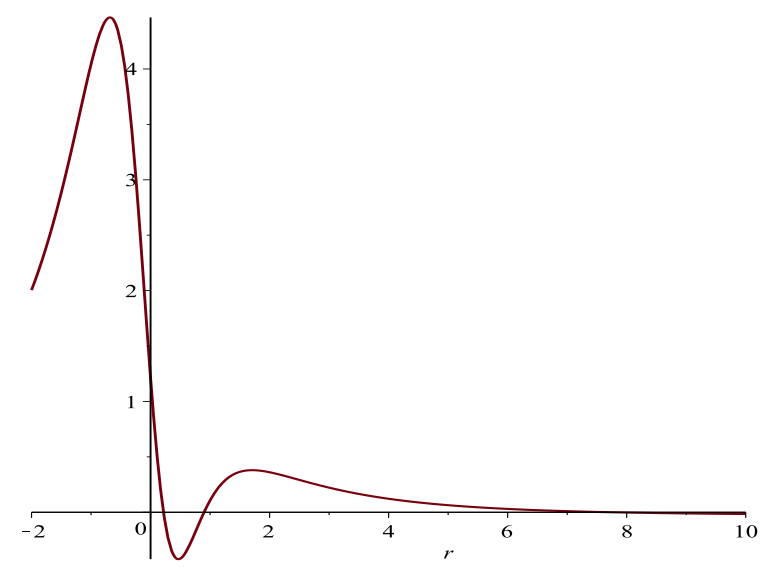

FIG. 1: Radial potential corresponding to $n=$ $1, m=.4, l=2.8, b=.3$ with two maxima and two minima. The rightest one at $r=16.23395$, $U=-0.2383 e-1$, not seen in this scale, is shown separately in Fig. 2.

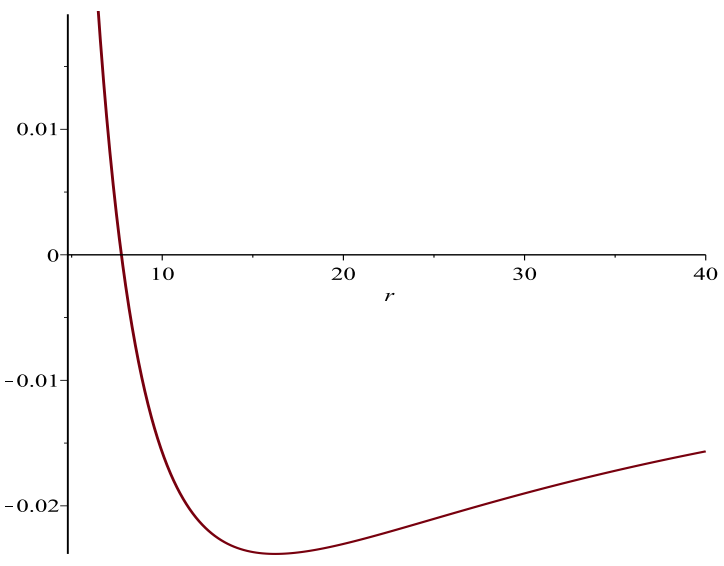

FIG. 2: The Newtonian potential well region of the potential Fig. 1 with higher resolution.

with the effective potential

$$
U(r)=\varepsilon \frac{2 m r+n^{2}-\alpha^{2}}{\rho^{2}}+\frac{l^{2}\left[(r-m)^{2}+b^{2}\right]}{\rho^{4}}
$$

with $\alpha^{2}=b^{2}+m^{2}=e^{2}-n^{2}$. Its derivative generically can presented as $U^{\prime}=P_{4}(r) / \rho^{6}$, where $P_{4}(r)$ is a polynomial of fourth order in $r$, which, depending on the parameter values, may have four real roots or two real roots. In the cases $m=0$ or $\varepsilon=0, P_{4}(r)$ degenerates to a polynomial of third order with three or one real roots.

In the case of timelike geodesics $(\varepsilon=-1)$, for $m \neq 0$ the potential is asymmetric under reflection $r \rightarrow-r$. We will assume $m>0$ which corresponds to a positive mass from the point of view of an observer at $r=+\infty$ and negative mass for an observer at $r=-\infty$. In the generic case one maximum is at negative $r$ while at $r>0$ there are two minima with a local maximum between, or only one minimum. A typical potential with four extrema is shown in Figs. 1-2. The case with only two extrema is illustrated in Fig. 3. Remarkably, the potential for zero orbital momentum $l=0$, generically has two extrema: a minimum at $r=r_{+}$and a maximum at $r=r_{-}$(see Fig. 4),

$$
r_{ \pm}=\frac{e^{2}-2 n^{2} \pm \sqrt{e^{4}-4 n^{2} b^{2}}}{2 m}
$$

corresponding to stable or unstable equilibrium positions. The potential for the massless wormhole $m=0$ is symmetric under reflection $r \rightarrow-r$, and so has an extremum at $r=0$, and two 


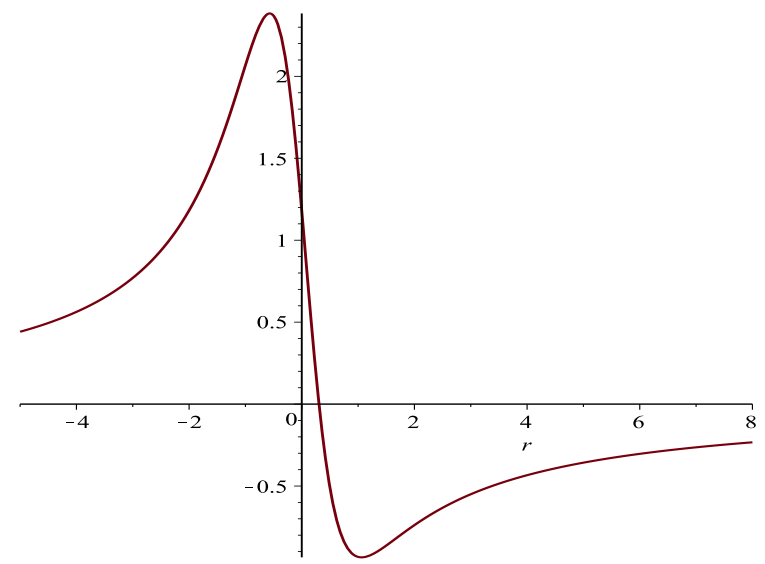

FIG. 3: The potential with two extrema for nonzero orbital momentum $(n=m=l=1, b=.3)$.

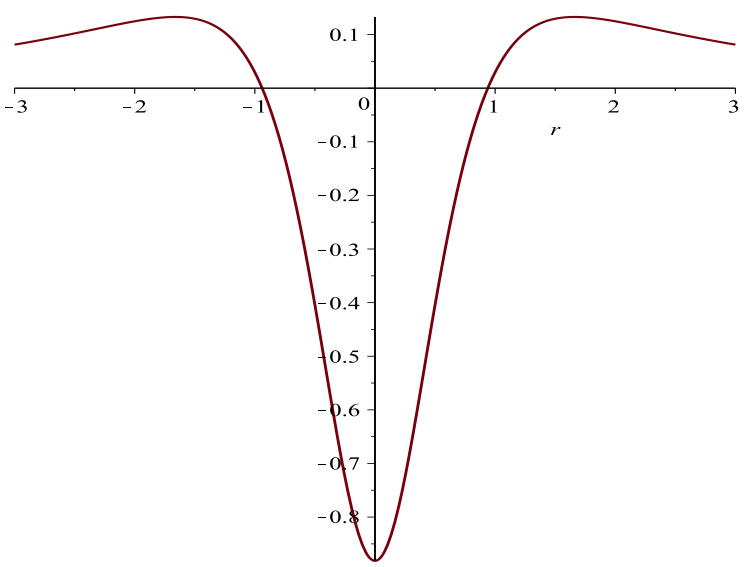

FIG. 5: The generic potential for zero mass $m=$ 0 with three extrema and $n=1, l=1, b=.2$

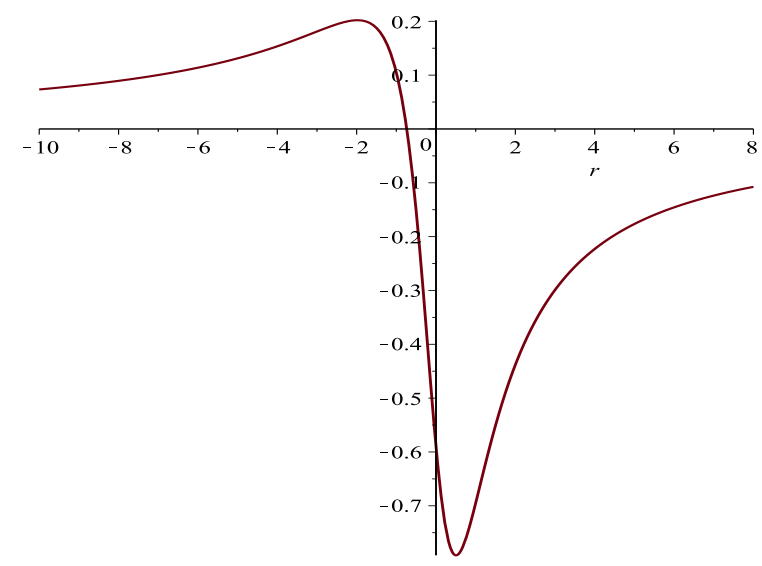

FIG. 4: The potential for zero orbital momentum $l=0$ and $n=1, m=.4, b=.5$. For $l=0$ the extrema can be found analytically by solving a quadratic equation.

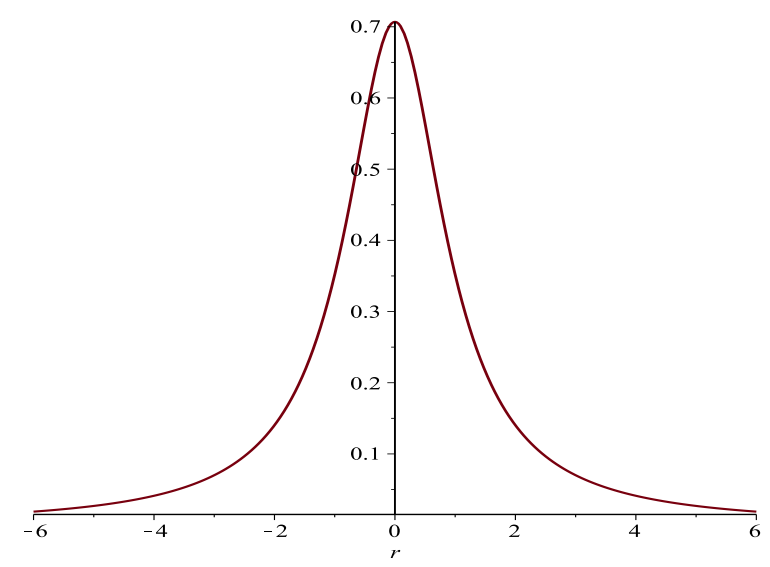

FIG. 6: Massless wormhole potential with one maximum $(m=0, l=0.1, n=1, b=1.3)$.

other extrema at $r= \pm r_{0}$ :

$$
r_{0}^{2}=\frac{\left(2 b^{2}-n^{2}\right) l^{2}+n^{2}\left(b^{2}-n^{2}\right)}{n^{2}-b^{2}-l^{2}},
$$

provided $0<n^{2}-b^{2}<l^{2}$ and $l^{2} \leq n^{2}\left(n^{2}-b^{2}\right) /\left(2 b^{2}-n^{2}\right)\left(\right.$ if $\left.4 b^{2}>n^{2} / 2\right)$.

The potential for null geodesics $\varepsilon=0$ is positive definite (Figs. 8-10). It has either one, or three extrema. In the massless case $m=0$ there is an extremum at $r=0$, and two other extrema at $r= \pm r_{1}$ with

$$
r_{1}^{2}=n^{2}-2 b^{2}
$$

provided $n^{2}>2 b^{2}$. In this case there is a stable circular photon orbit at the wormhole throat 


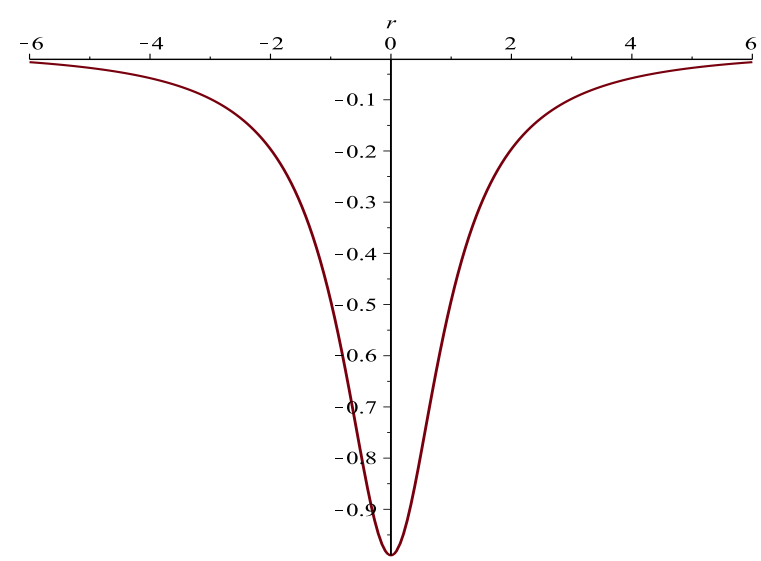

FIG. 7: Massless wormhole potential with one minimum $(m=0, l=.1, n=1, b=0.1$.

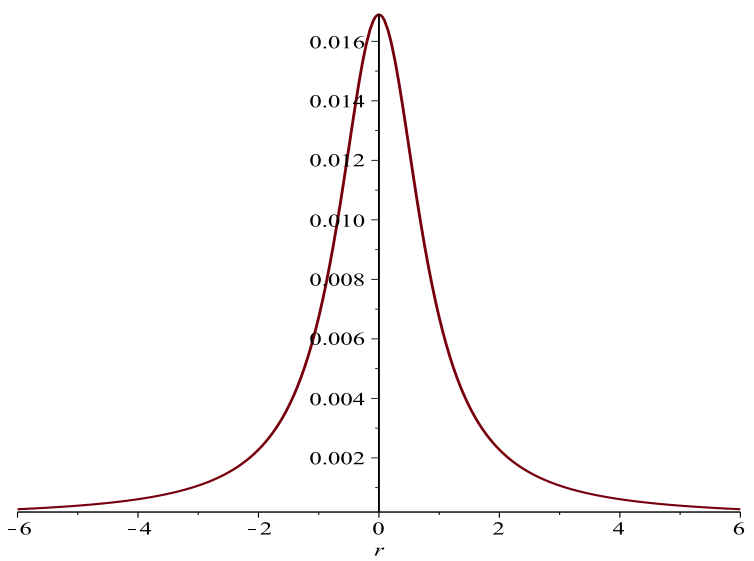

FIG. 9: Massless wormhole potential for null geodesics with one maximum $(m=0, l=$ $0.1, n=1, b=1.3)$.

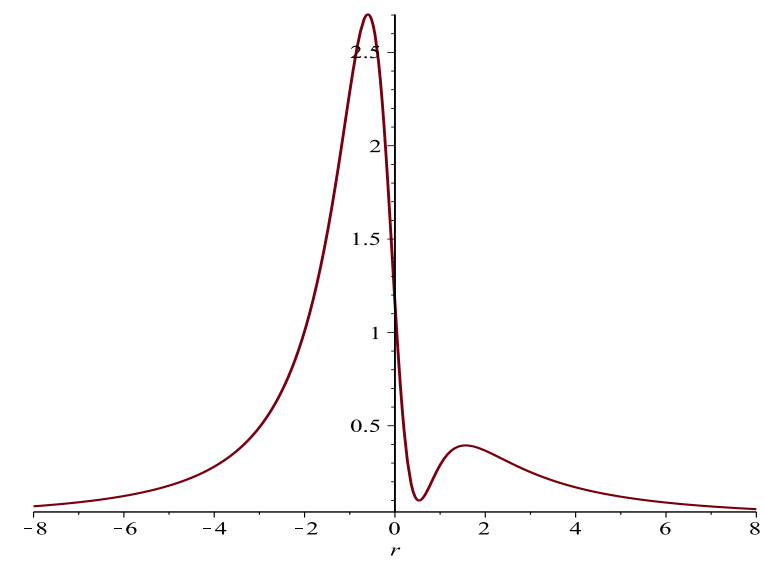

FIG. 8: Generic potential for null geodesics with three extrema $(n=1, m=1 / 2, l=2, b=.2)$.

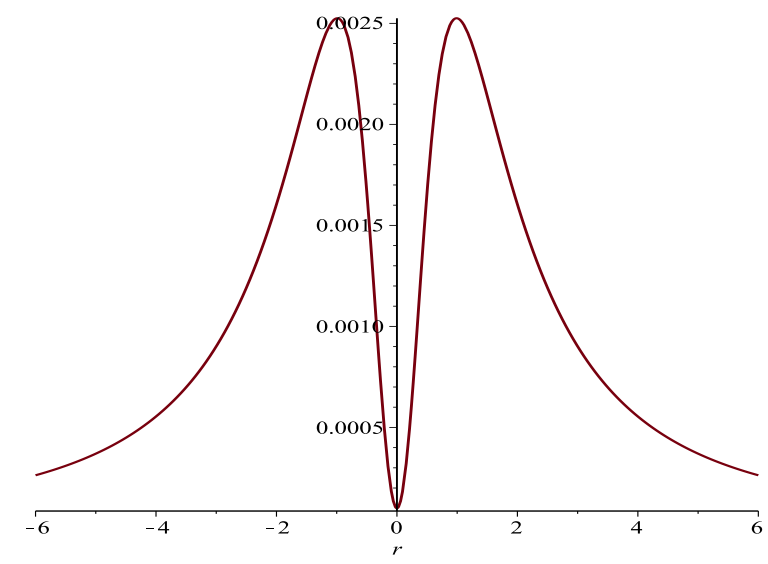

FIG. 10: Massless wormhole potential for null geodesics with three extrema $(m=0, l=.1, n=$ $1, b=0.1)$

$r=0$, with areal radius $n$, and unstable photon orbits at $r= \pm r_{1}$. The purely radial motion $(l=0)$ of the photons is free.

By following the procedure of [19], exact solutions for $r(\lambda)$ can be obtained in terms of Weirstrass elliptic functions. Here we will only discuss the qualitative radial motion for timelike or null geodesics sampling the wormhole $\left(b^{2}>0\right)$ geometry, and consider special limiting cases of interest. We shall work in the rotated coordinate frame in which the $z$ axis is along $\vec{J}$, so that the polar angle $\theta$ is constant. 


\section{B. Timelike geodesics $(\varepsilon=-1)$}

\section{Scattering states}

The effective potential is for $m>0$ negative for large positive $r$ and positive for large negative $r$, and so presents at least a maximum and a minimum, with $r_{\min }>r_{\max }$, and possibly also a secondary maximum and a secondary minimum. A test particle coming from infinity with $E^{2} \geq 1$ will first be accelerated in the potential well around $r_{\text {min }}$, before either being reflected (scattered) by the potential barrier or, if its energy is high enough, being transmitted through the wormhole neck $r=r_{\max }$ to the second asymptotic sheet $r \rightarrow-\infty$.

Combining this radial motion with the angular motion previously discussed leads to a trajectory which spirals around the polar axis $\vec{J}$ at a constant angular velocity $J$ with respect to $\lambda$. The definition (3.13) of $\lambda$ together with (4.1) leads to the relation

$$
d \lambda=\frac{d r}{\left(r^{2}+n^{2}\right)\left[E^{2}-1-U(r)\right]^{1 / 2}} .
$$

Putting $\beta^{2}=E^{2}-1$, it follows that the geodesic range of $\lambda$ is given by the finite integrals

$$
\Delta \lambda_{\text {refl }}=2 \int_{r_{0}}^{\infty} \frac{d r}{\left(r^{2}+n^{2}\right)\left[\beta^{2}-U(r)\right]^{1 / 2}}
$$

in the case of reflexion (with $r_{0}$ the turning point, $U\left(r_{0}\right)=\beta^{2}$ ), or

$$
\Delta \lambda_{\text {trans }}=\int_{-\infty}^{\infty} \frac{d r}{\left(r^{2}+n^{2}\right)\left[\beta^{2}-U(r)\right]^{1 / 2}}
$$

in the case of transmission (if $\beta^{2}>\beta_{0}^{2}=U_{\max }$ ). Defining as usual the impact parameter by $a=l / \beta$, the cross-section for transmission is

$$
\sigma_{\text {trans }}=\pi a_{0}^{2}=\frac{\pi l^{2}}{U_{\max }} .
$$

In the limiting intermediate case $\beta^{2}=\beta_{0}^{2}$, the integral (4.7) with the lower bound $r_{\max }$ is logarithmically divergent, and the trajectory, attracted by the unstable circular orbit $r=r_{\max }$, spirals indefinitely towards the wormhole neck. In the present paper, we will only evaluate these integrals for two special cases of interest: the non-relativistic limit, and the small-NUT limit.

The non-relativistic limit is defined by $m=0$ and $b^{2}=n^{2}$ (so that $f(r)=1$ ) and $\beta^{2} \ll 1$. In this case,

$$
U(r)=l^{2} /\left(r^{2}+n^{2}\right)
$$

leads to $r_{0}^{2}=a^{2}-n^{2}$, with

$$
\Delta \lambda_{\mathrm{refl}}=\frac{2}{\beta} \int_{r_{0}}^{\infty} \frac{d r}{\left[\left(r^{2}+n^{2}\right)\left(r^{2}-r_{0}^{2}\right)\right]^{1 / 2}}=\frac{2}{l} K\left(\frac{n}{a}\right),
$$


where $K$ is the complete elliptic integral of the first kind. In the frame $\psi=0$, the corresponding total angular variation is

$$
\Delta \varphi_{\mathrm{refl}}=J \Delta \lambda_{\mathrm{refl}} \simeq \frac{2}{\sin \theta} K\left(\frac{\beta}{2} \cot \theta\right),
$$

where we have used $E \simeq 1$ for small $\beta$. If $l \simeq 2 n \tan \theta$ is held fixed in the non-relativistic limit (corresponding to large impact parameters $a$ ), (4.12) reduces (using $K(0)=\pi / 2$ ) to the finite result $\Delta \varphi_{\text {refl }} \simeq \pi / \sin \theta$. If on the other hand the impact parameter $a$ is held fixed (of the order of $n$ ), then the cone angle $\theta$ goes to zero, and the test particle spirals around the polar axis many times before being scattered back to infinity.

Similarly, in the case of transmission through the wormhole $\left(a<a_{0}=n\right)$,

$$
\Delta \lambda_{\text {trans }}=\frac{2}{\beta} \int_{0}^{\infty} \frac{d r}{\left[\left(r^{2}+n^{2}\right)\left(r^{2}+n^{2}-a^{2}\right)\right]^{1 / 2}}=\frac{2}{\beta n} K\left(\frac{a}{n}\right) .
$$

Again, if $\beta \ll 1$ the cone angle $\theta \simeq \beta a / 2 n$ is very small, and the test particle makes a finite but large number of turns

$$
\frac{\Delta \varphi_{\text {trans }}}{2 \pi} \simeq \frac{2}{\pi \beta} K\left(\frac{a}{n}\right)
$$

along its path through the wormhole.

Remarkably, the number of turns remains large,

$$
\frac{\Delta \varphi_{\text {trans }}}{2 \pi} \simeq \frac{1}{\beta}
$$

in the limit of a purely radial trajectory, $l=0(a=0)$. This fact, which results from the conservation of total angular momentum (3.6) $(\vec{J}=\vec{S}$ in the case of purely radial motion, $\vec{L}=0$ ), is of course not possible for a classical point test particle, but makes sense if one considers rather a small extended solid test body. For instance, (4.15) means that a tennis ball, of radius small before the wormhole throat radius $n$, will spin around, with an angular velocity

$$
\frac{d \varphi}{d \tau}=\frac{2 n E}{r^{2}+n^{2}}
$$

which is maximum at the wormhole throat $r=0$, making $\beta^{-1}$ turns while going straight through the wormhole from $r=+\infty$ to $r=-\infty$.

Let us emphasize that this uniform rotation of an extended test body in free radial fall does not depend on the direction of this radial motion, which generically does not coincide with that of the Misner string. From (3.19) and (3.25), the angular motion along a parallel $\mathcal{C}$ is a uniform rotation, with proper angular velocity $J /\left(r^{2}+n^{2}\right)$. In the limit of a purely radial motion, $l \rightarrow 0$, the parallel $\mathcal{C}$ contracts to a point $\theta=$ constant, $\varphi=$ constant, while the uniform rotation velocity retains the finite value $2 n E /\left(r^{2}+n^{2}\right)$. 
The small-NUT limit is motivated by the non-observation of NUT charge in nature. It is defined by $n^{2} \ll m^{2}$ and $n^{2} \ll b^{2}$, so that $\alpha^{2} \simeq e^{2}$. In this limit the effective potential (4.2) can be approximated by

$$
U(r) \simeq \frac{-2 m r^{3}+\left(e^{2}+l^{2}\right) r^{2}-2 m l^{2} r+e^{2} l^{2}}{\left(r^{2}+n^{2}\right)^{2}},
$$

and its derivative by

$$
U^{\prime}(r) \simeq \frac{2 r\left[m r^{3}-\left(e^{2}+l^{2}\right) r^{2}+3 m l^{2} r-2 e^{2} l^{2}\right]}{\left(r^{2}+n^{2}\right)^{3}}
$$

Obviously $r=0$ is a maximum of $U(r)$, and an absolute maximum because $U(0)-U(r) \geq 0$ in the small NUT limit:

$$
\beta_{0}^{2}=U_{\max } \simeq U(0) \simeq \mid \begin{array}{cc}
\frac{e^{2} l^{2}}{n^{4}} & (l \neq 0), \\
\frac{e^{2}}{n^{2}} & (l=0) .
\end{array}
$$

We first consider the case of reflexion $\left(\beta^{2}<\beta_{0}^{2}\right)$, which we will discuss only in the small-velocity limit $(\beta \rightarrow 0)$. In this limit $l=\beta a$ can be neglected (purely radial motion), so that

$$
\beta^{2}-U \simeq-U \simeq \frac{2 m r-e^{2}}{r^{2}+n^{2}}
$$

with $r_{0} \simeq e^{2} / 2 m$. The evaluation of the integral (4.7) yields a small, but non-vanishing

$$
\frac{\Delta \varphi_{\mathrm{refl}}}{2 \pi} \simeq \frac{2 n}{e}
$$

Transmission through the wormhole neck occurs if $\beta^{2}>\beta_{0}^{2}$, i.e. in the extreme-relativistic case. The cone angle is given in this case by $\tan \theta \simeq a / 2 n<n / 2 e$. In the extreme-relativistic limit we can approximate

$$
\beta^{2}-U \simeq \beta^{2}-\frac{\beta_{0}^{2} n^{4}}{\left(r^{2}+n^{2}\right)^{2}}=\beta^{2}\left[1-\frac{e^{2} a^{2}}{\left(r^{2}+n^{2}\right)^{2}}\right] .
$$

The evaluation of the integral (4.8) yields

$$
\Delta \varphi_{\mathrm{trans}} \simeq \frac{4 n}{\sqrt{n^{2}+e a}} K\left(\sqrt{\frac{2 e a}{n^{2}+e a}}\right) .
$$

For very small impact parameters (corresponding to finite anglar momenta $l=\beta a$ ) $a \ll n^{2} / e$, (4.23) reduces to the finite result $\Delta \varphi_{\text {trans }} \simeq 2 \pi$. Again, this remains valid in the limit of a purely radial trajectory, so that a small extended test body falling straight through the wormhole will at the same time spin around (at a maximum proper angular velocity $d \varphi / d \tau \simeq 2 \beta / n$ ), making exactly one turn while going from $r=+\infty$ to $r=-\infty$. 


\section{Bound states}

Bound states in the wormhole Brill geometry have some unusual features with respect to the typical black hole situation. The generic potential (Fig. 1) for massive particles has two potential wells: one is at negative $U$ in the far region with small binding energies (non-relativistic Newtonian orbits) shown in Fig. 2. Another, relativistic potential well closer to the throat, extends to positive energies, being separated from escape by a potential barrier (Fig. 1) with larger binding energies. One has therefore oscillating bound orbits between the two turning points of two types: Newtonian, and relativistic. At the corresponding minima one has two stable circular orbits. If two roots of the equation $U^{\prime}(r)=0$ are complex, the potential has a simpler shape with only two extrema, Fig. 3, the minimum (potential well) being in the $r>0$ region, and the maximum at some $r<0$. As it is seen from this figure, the potential well is deep and relativistic, the Newtonian well being absent in this case.

For zero orbital momentum $l=0$, the potential reduces to the simple form

$$
U(r)=\frac{(r-m)^{2}+b^{2}}{\rho^{2}}-1
$$

with a minimum and a maximum given by Eq. (4.3). One can observe that the the shape of the potential for $l=0$ (Fig. 3) is qualitatively similar to that for $l \neq 0$ with two extrema (Fig. 4). Indeed for $l=0$ we still have a non-zero total angular momentum $J=2 n E$. The bound

orbits are oscillating between two turning points with relativistic velocities. The test particle can remain at rest at the minimum at $r=r_{+}$provided its squared energy

$$
E_{0}^{2}=\frac{e^{2}-\sqrt{e^{4}-4 n^{2} b^{2}}}{2 n^{2}}
$$

is positive, i.e. if $b^{2}>0$. However for $n \neq 0$, a small test body at the equilibrium position $r=r_{0}$ will spin around with angular velocity $\omega_{0}=2 n E_{0} /\left(r_{0}^{2}+n^{2}\right)$. In the small-NUT limit, these $l=0$ equilibrium values reduce to

$$
r_{0} \simeq \frac{e^{2}}{m}, \quad E_{0} \simeq \frac{b}{e}, \quad \omega_{0} \simeq \frac{2 n b m^{2}}{e^{5}}
$$

The potential is particularly simple and reflection-symmetric in the case of the massless wormhole $m=0$. Depending on the parameter values, there could be three different cases: a potential well centered at the throat extending to positive energy values and separated from the scattering region by potential barriers (Fig. 5), a pure potential barrier centered at the throat (Fig. 6) and a purely negative potential well at the throat (Fig. 7). In the first and the last cases one thus has stable bound orbits oscillating around the throat, including circular ones located exactly at the throat. 
Another simple case is the near-extreme limit $b^{2} \ll m^{2}$. The effective potential (4.2) has its absolute minimum at $r=m$ (the extreme RN horizon radius) for $b^{2}=0\left(e^{2}=m^{2}+n^{2}\right)$. So for $b^{2}$ small and positive, there are stable circular orbits at $r=m+\mathrm{O}\left(b^{2}\right)$, with energy $E_{l}$ and proper angular velocity $\omega_{l}=d \varphi / d \tau$

$$
E_{l}^{2} \simeq b^{2} \frac{e^{2}+l^{2}}{e^{4}}, \quad \omega_{l} \simeq \frac{l}{e^{2}} .
$$

\section{Null geodesics $(\varepsilon=0)$}

The effective potential for null geodesics

$$
U(r)=\frac{l^{2}\left[(r-m)^{2}+b^{2}\right]}{\left(r^{2}+n^{2}\right)^{2}}
$$

is shown in Figs. 8-10. The discussion of the scattering of light parallels that for timelike geodesics. Of special interest is the scattering angle $\Delta \varphi_{\text {refl }}=J \Delta \lambda_{\text {refl }}$, where $\Delta \lambda_{\text {refl }}$ is given by (4.7) with $\beta=E$, and $J^{2}=\left(4 n^{2}+a^{2}\right) E^{2}$. This can be computed analytically in the special case $m=0$, with the result

$$
\begin{aligned}
& \Delta \varphi_{\text {refl }}=2 \sqrt{\frac{4 n^{2}+a^{2}}{\alpha^{2}}} K\left(\sqrt{1-\frac{r_{0}^{2}}{\alpha^{2}}}\right) \quad \text { for } \quad a^{2}>\frac{n^{4}}{b^{2}}, \\
& \Delta \varphi_{\text {refl }}=2 \sqrt{\frac{4 n^{2}+a^{2}}{r_{0}^{2}}} K\left(\sqrt{1-\frac{\alpha^{2}}{r_{0}^{2}}}\right) \quad \text { for } \quad n^{2}>2 b^{2} \text { and } 4\left(n^{2}-b^{2}\right)<a^{2}<\frac{n^{4}}{b^{2}},
\end{aligned}
$$

with

$$
\alpha^{4}=a^{2}\left[a^{2}+4\left(b^{2}-n^{2}\right)\right], \quad r_{0}^{2}=\frac{\alpha^{2}+a^{2}-2 n^{2}}{2} .
$$

In the non-relativistic case $b^{2}=n^{2}$, this reduces for $a>n$ to

$$
\Delta \varphi_{\mathrm{refl}}=2 \sqrt{1+\frac{4 n^{2}}{a^{2}}} K\left(\frac{n}{a}\right)=\frac{2}{\sin \theta} K\left(\frac{1}{2} \cot \theta\right)
$$

where $\tan \theta>1 / 2$, while light rays with small impact parameters $a<n(\tan \theta<1 / 2)$ end up in the second asymptotic sheet $(r \rightarrow-\infty)$ with

$$
\Delta \varphi_{\mathrm{trans}}=\frac{4}{\cos \theta} K(2 \tan \theta)
$$

Let us also note the existence, in the near-extreme limit $b^{2} \ll m^{2}$, of circular null orbits at $r \simeq m$ (as in the case of massive particles) with energy

$$
E_{l}^{2} \simeq b^{2} \frac{l^{2}}{e^{4}}
$$




\section{CHARGED PARTICLE MOTION}

Consider now the non-geodesic motion of a charged particle with mass $m_{c}$ and charge $q_{c}$. It is convenient to work with the covariant Lorentz equations of motion:

$$
\left(\dot{x}^{\nu} g_{\mu \nu}\right)^{\cdot}-\frac{1}{2} \partial_{\mu} g_{\nu \lambda} \dot{x}^{\nu} \dot{x}^{\lambda}=\kappa F_{\mu \nu} \dot{x}^{\nu}
$$

where $\kappa=q_{c} / m_{c}$. Since $A_{\mu}$ has only $\mu=a=(t, \varphi)$ components depending only on $r$, $\theta$, one has for $\mu=a$

$$
F_{a \nu} \dot{x}^{\nu}=-\dot{A}_{a}
$$

while for the remaining $\mu=i=(r, \theta)$ components

$$
F_{i \nu} \dot{x}^{\nu}=\partial_{i} A_{a} \dot{x}^{a}
$$

The equations of motion thus split into two groups. The first group of equations do not contain the metric derivative term and combine to a total derivative

$$
\left(\dot{x}^{\nu} g_{a \nu}+\kappa A_{a}\right)^{\cdot}=0
$$

which can be integrated as before by introducing two integrals of motion $E, J_{z}$

$$
\begin{aligned}
& f(r)(\dot{t}-2 n(\cos \theta+C) \dot{\varphi})-\kappa \Phi(r)=E \\
& \dot{x}^{\nu} g_{\varphi \nu}+\kappa A_{\varphi}=J_{z}+2 n C
\end{aligned}
$$

the second one being equivalent to (3.2). In the second group it is enough to consider only the $\theta$ component, taking the normalization condition (3.4) as a constraint. The $i=\theta$ component reads

$$
\left[\left(r^{2}+n^{2}\right) \dot{\theta}\right]^{\cdot}=\frac{1}{2} g_{a b, \theta} \dot{x}^{a} \dot{x}^{b}+\kappa A_{a, \theta} \dot{x}^{a}=g_{t \varphi, \theta} \dot{t} \dot{\varphi}+\frac{1}{2} g_{\varphi \varphi, \theta} \dot{\varphi}^{2}+\kappa A_{\varphi, \theta} \dot{\varphi} .
$$

Eliminating $\dot{t}$ from (5.5), we find that the $\kappa$-terms cancel, and we are left precisely with the equation

$$
\left[\left(r^{2}+n^{2}\right) \dot{\theta}\right]^{\cdot}=\left(r^{2}+n^{2}\right) \sin \theta \cos \theta \dot{\varphi}^{2}-2 n E \sin \theta \dot{\varphi}
$$

identical to (3.3). So the angular equations of motion are the same as in the case of a neutral particle, leading to orbits which lie on a "cone" of half-angle $\arctan (2 n E / l$ ) (with $l$ the orbital angular momentum), while in the equation for the time evolution (3.1) and in the radial equation (3.12) the constant $E$ must be replaced by $E+\kappa \Phi(r)$ leading to

$$
\dot{r}^{2}+f(r)\left[\frac{l^{2}}{r^{2}+n^{2}}+1\right]=(E+\kappa \Phi(r))^{2},
$$


where we took $\varepsilon=-1$ for massive, charged particles. This can be rewritten as

$$
\dot{r}^{2}+W(r)=0, \quad \text { where } \quad W(r)=f(r)\left[\frac{l^{2}}{r^{2}+n^{2}}+1\right]-(E+\kappa \Phi(r))^{2} .
$$

The quantity $W(r)$ however is not convenient for the role of the effective radial potential since it depends quadratically on $E$ and contains unphysical negative energies. To correctly introduce the radial potential we have to present this equation in the factorized form

$$
\dot{r}^{2}=\left(E-V_{+}(r)\right)\left(E-V_{-}(r)\right)
$$

where

$$
V_{ \pm}(r)=-\kappa \Phi \pm \sqrt{f\left(1+\frac{l^{2}}{r^{2}+n^{2}}\right)},
$$

so that for a particle at rest $\dot{r}=0$ the correct branch is ${ }^{1} E=V_{+}$. Moreover, the quantity $E+\kappa \Phi(r)$ is equal to the kinetic energy, it has therefore to be strictly positive (recall that $f>0$ everywhere for the wormhole):

$$
E+\kappa \Phi(r)=\sqrt{\dot{r}^{2}+f(r)\left[\frac{l^{2}}{r^{2}+n^{2}}+1\right]}>0 .
$$

It follows that $E>V_{-}$always, so the particle's motion corresponds to $E>V_{+}$. Also, the conditions for circular orbits $W=0=W^{\prime}$ are implied by the more physical conditions $V_{+}=$ $V_{+}^{\prime}=0$ which do not contain unphysical negative energies. So we can consider $V_{+}$as a correct radial potential.

To compare with the results of [23] for the motion of charged particles in the dyonic ReissnerNordström spacetime, one must first gauge transform our potential $\Phi(r)$ to a potential vanishing at infinity $\Phi_{0}(r)$. This transformation and the associated shift in the integration constant $E$ are

$$
\Phi(r)=\Phi_{0}(r)+\frac{p}{2 n}, \quad E=E_{0}-\frac{\kappa p}{2 n} .
$$

Taking the NUT charge to zero, we find that the orbit of a charged particle in the dyonic Reissner-Nordström spacetime lies on a cone of half-angle $\arctan (\kappa p / l)$, in agreement with the results of $[23]$.

\footnotetext{
${ }^{1}$ Recall that in special relativity the total energy of a charge in the potential $\varphi$ (note that $\Phi$ is defined with an opposite sign to $\varphi$ ) satisfying $(E-e \varphi)^{2}=m^{2}+\mathbf{p}^{2}$ has to be solved with the sign plus: $E=e \varphi+\sqrt{m^{2}+\mathbf{p}^{2}}$. In our case the mass is absorbed by the affine parameter on the worldline, so $m=1$.
} 


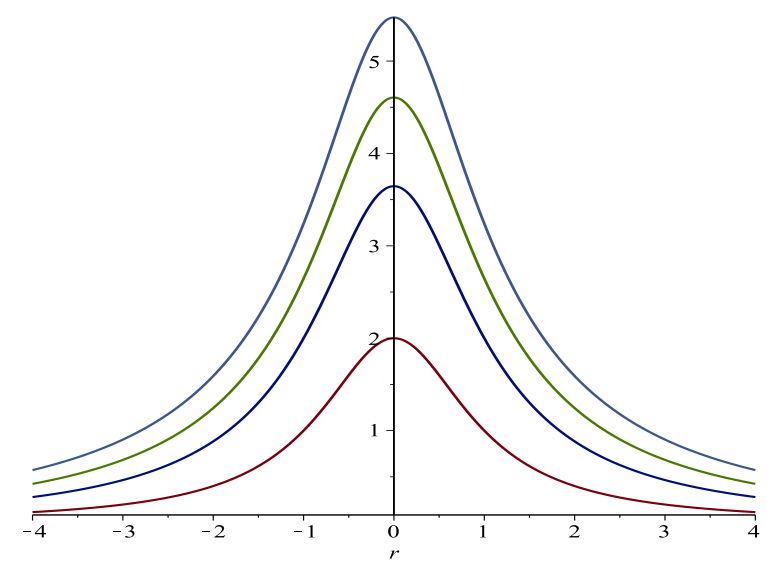

FIG. 11: Radial potential for charged particle motion in the wormhole with $m=0, q=0, p^{2}=$ $2 n^{2}$ in the case of positive $\kappa$ for different angular momenta $l^{2}\left(\kappa=\sqrt{2}, l^{2}=19,12,6,0\right.$ decreasing from top to bottom).

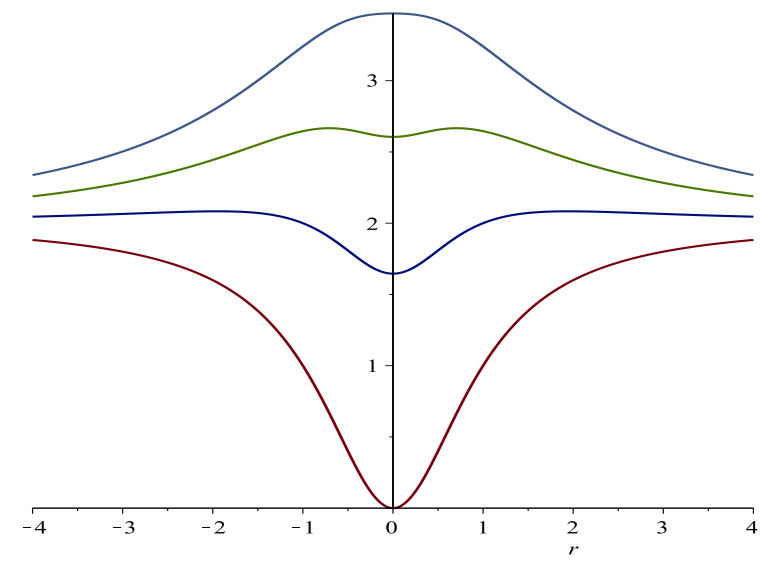

FIG. 12: Radial potential for negative $\kappa(=-\sqrt{2})$ and the same values of $l^{2}$ from top to bottom.

\section{A. Motion in the case $f=1$}

We restrict to the particularly simple case of the massless wormhole with only magnetic and NUT charges related so tha $f(r)=1$,

$$
m=0, \quad q=0, \quad p=\sqrt{2} n,
$$

leading to

$$
V_{+}=-\frac{\kappa}{\sqrt{2}} \frac{r^{2}-n^{2}}{r^{2}+n^{2}}+\sqrt{1+\frac{l^{2}}{r^{2}+n^{2}}} .
$$

The potential $V_{+}$is symmetrical under reflection $r \rightarrow-r$. For $\kappa>0$ it is maximum at the wormhole throat $r=0$ and monotonically decreases with growing $|r|$ (Fig. 11). The maximal value depends on the orbital momentum as follows:

$$
V_{0} \equiv V_{+}(0)=\frac{\kappa}{\sqrt{2}}+\sqrt{1+\frac{l^{2}}{n^{2}}} .
$$

For $\kappa<0$ the shape of the potential is more diverse, as shown in Fig. 12. In the region I:

$$
\frac{l^{2}}{n^{2}}<2 \sqrt{2}|\kappa|
$$

the potential has a local minimum at $r=0$. With growing $l^{2}$ the local minimum goes up and in the region $\mathrm{II}$ :

$$
2 \sqrt{2}|\kappa|<\frac{l^{2}}{n^{2}}<4 \kappa^{2}+2|\kappa| \sqrt{2\left(1+2 \kappa^{2}\right)}
$$


local maxima develop on both sides of the throat, with the positions

$$
r_{ \pm}= \pm n \sqrt{\frac{8 \kappa^{2} n^{2}\left(n^{2}+l^{2}\right)-l^{4}}{l^{4}-8 \kappa^{2} n^{4}}}
$$

Finally, in the region III,

$$
\frac{l^{2}}{n^{2}}>\left(4 \kappa^{2}+2|\kappa| \sqrt{2\left(1+2 \kappa^{2}\right)}\right)
$$

the two local maxima at $r=r_{ \pm}$disappear, while the local minimum at $r=0$ turns into an absolute maximum.

With the potentials shown in Fig. 11, 12 one deduces the following characterization of the orbits. For $\kappa>0$ one has orbits traversing the wormhole throat for $E>V_{0}$ corresponding to the top of the potential barrier in Fig. 11. The orbits with $E<V_{0}$ correspond to scattering on the wormhole. For $\kappa<0$ in the parameter region III the situation is the same. In region II one has in addition bound states between the turning points inside the potential well between the local maxima. The same energies correspond to scattering orbits reflected on the exterior sides of the barriers. The region I contains bound orbits inside the well and traversing orbits for the energies above the wells. These can be analyzed in analogy with Sec. 4, we leave it for future work.

The circular orbits at the throat $r=0$ (with areal radius $n$ ) exist for both signs of $\kappa$. These will be interesting for analysis of non-causality in the next section. They are unstable for positive $\kappa$ and negative $\kappa$ in the region III, but stable for negative $\kappa$ in the regions I and II. The corresponding gauged energy $E=V_{+}(0)+\kappa / \sqrt{2}$ is positive for positive $\kappa$, but it is negative for negative $\kappa$ if

$$
\frac{l^{2}}{n^{2}}<2 \kappa^{2}-1 \quad(|\kappa|>1 / \sqrt{2}) .
$$

In the case of negative $\kappa$, two other unstable circular orbits exist in region II at $r=r_{ \pm}$ corresponding to the kinetic energy

$$
E+\kappa \Phi\left(r_{ \pm}\right)=\frac{l^{2}}{2 \sqrt{2}|\kappa| n^{2}}
$$

and positive total energy

$$
E=\frac{|\kappa|}{\sqrt{2}}\left(\frac{l^{2}}{4 \kappa^{2} n^{2}}+1+\frac{2 n^{2}}{l^{2}}\right)
$$

\section{CAUSALITY}

The ADM form of the metric(2.1) is

$$
d s^{2}=-\frac{f\left(r^{2}+n^{2}\right) \sin ^{2} \theta}{\Sigma} d t^{2}+f^{-1} d r^{2}+\left(r^{2}+n^{2}\right) d \theta^{2}+\Sigma\left(d \varphi+\frac{2 n f(\cos \theta+C)}{\Sigma} d t\right)^{2}
$$


with

$$
\Sigma(r, \theta)=\left(r^{2}+n^{2}\right) \sin ^{2} \theta-4 n^{2} f(\cos \theta+C)^{2} .
$$

For $f(r)>0$ (which is always the case for the wormhole Brill solution with $b^{2}>0$ ), $\Sigma$ becomes negative, and closed timelike curves (CTCs) appear, in a neighborhood of the Misner string given by $\Sigma(r, \theta)<0$. The surface $\Sigma(r, \theta)=0$ bounding this CTC neighborhood is a causal singularity of the spacetime, where the signature of the spacetime changes from $(-+++)$ outside to $(+++-)$ inside. This singularity is, just as the Misner string itself, completely transparent to geodesic motion. Nevertheless, the occurrence of CTCs in a spacetime is usually considered

to violate causality $[3,24]$. An observer traveling around such a CTC would eventually return to his original spacetime position after a finite proper time lapse, thus opening the possibility for time travel. However, unless this observer is freely falling, such a CTC travel would necessarily involve accelerations generated e.g. by rocket engines. One can argue that the back-reaction of these matter accelerations on the spacetime geometry will deform it in such a way that chronology will ultimately be preserved. If this reasoning is correct, in vacuum gravity causality violation can only occur in spacetimes with closed timelike geodesics (CTGs), or possibly closed null geodesics (CNGs). However we deal here with a charged solution, so we must also consider the worldlines of charged particles.

\section{A. No closed timelike or null geodesics}

We now show that there are no closed timelike or null geodesics in the Brill spacetimes with $|C| \leq 1$. Closed geodesics (or self-intersecting geodesics) occur if after a finite lapse of affine parameter $\tau$ or of Mino time $\lambda$, all the coordinates take again the same values (modulo $2 \pi$ for the azimuthal angle). So the perimeter of the geodesic must be an integer multiple of the $\lambda$-period $2 \pi / J$ of the angular motion. From (3.36), during this period,

$$
\Delta t_{\theta} \geq 4 \pi n\left(\frac{2 n E}{J}-1\right)
$$

for $|C| \leq 1$. Also, from (3.32) and (3.12),

$$
\frac{d t_{r}}{d \lambda}=\frac{E\left(r^{2}+n^{2}\right)}{f(r)} \geq E^{-1}\left[l^{2}-\varepsilon\left(r^{2}+n^{2}\right)\right] \geq E^{-1}\left[l^{2}-\varepsilon n^{2}\right],
$$

leading to

$$
\Delta t_{r} \geq \frac{2 \pi}{E J}\left[l^{2}-\varepsilon n^{2}\right]
$$

over the same period. Adding the two together, we obtain

$$
\Delta t=\Delta t_{r}+\Delta t_{\theta} \geq \frac{2 \pi}{E}\left[J-2 n E-\varepsilon n^{2} / J\right] .
$$


For $\varepsilon=-1$ this is clearly positive definite. For $\varepsilon=0$, this can vanish only for $2 n E=J(l=0)$. But in this case $\Delta t_{\theta} \geq 0$, while $d t_{r} / d \lambda$, and thus also $\Delta t_{r}$, is positive definite. Thus, for $|C| \leq 1$ all timelike or null geodesics are causal (future directed).

The situation for $|C|>1$ is less obvious. We shall only discuss the case of null geodesics. As we shall show, 1) there are always CNGs in any given Brill spacetime if $|C|$ is large enough; 2) for any given value of $|C|>1$, there are Brill spacetimes with CNGs.

First, we observe that for $C>1$,

$$
\Delta t_{\theta}=4 \pi n\left[\frac{2 n E}{J}-|C|\right]
$$

for orbits which circle the South Misner string $\left(J_{z}<-2 n E\right)$. The same relation (6.7) holds for $C<-1$ in the case of orbits which circle the North Misner string $\left(J_{z}>2 n E\right)$. We wish to show that, if the orbit is closed, this negative angular contribution $(2 n E<J)$ can be exactly balanced by the positive radial contribution, leading to $\Delta t=0$ and thus to a closed geodesic. Depending on the values of the model parameters, two situations can lead to closed orbits with $\varepsilon=0$. Either $U(r)$ has a local minimum $U_{\min }>0$ between two local maxima, and there are bound states in the resulting potential well, leading to closed orbits if the periods of the radial and angular motion are commensurate (i.e. for a dense set of values of the energy). Or $U(r)$ has only a maximum, corresponding to an unstable circular orbit. Let us discuss these two cases separately.

In the first case, from (3.32) and (3.12),

$$
\frac{d t_{r}}{d \lambda}=l^{2} \frac{E}{U(r)} \leq l^{2} \frac{E}{U_{\min }} .
$$

It then follows that, over an angular period,

$$
\Delta t \leq 4 \pi n\left[\left(1+\frac{l^{2}}{4 n^{2} U_{\min }}\right) \frac{2 n E}{J}-|C|\right]<4 \pi n\left[1+\frac{l^{2}}{4 n^{2} U_{\min }}-|C|\right]
$$

vanishing for $|C|=1+l^{2} / 4 n^{2} U_{\text {min }}$ (which depends only on the wormhole parameters $(m, n, b)$ ). For higher values of $|C|$, the bound state null geodesics will be past directed.

In the case of a circular orbit of radius $r_{0}$ and energy $E_{0}=U\left(r_{0}\right)$ (circling the North or South Misner string as above),

$$
\frac{d t_{r}}{d \lambda}=\frac{l^{2}}{E_{0}}=\frac{J^{2}-4 n^{2} E_{0}^{2}}{E_{0}}
$$

leading to

$$
\Delta t=\frac{2 \pi}{E_{0}}\left[J-2 n E_{0}|C|\right]
$$


which vanishes for

$$
|C|=\frac{J}{2 n E_{0}}=\left(1+\frac{l^{2}}{4 n^{2} U\left(r_{0}\right)}\right)^{1 / 2}
$$

(value depending only on the wormhole parameters). So again in this case, for any given set of Brill parameters $(m, n, b)$, there will be CNGs for large enough $|C|$.

We now formulate the converse, and stronger, proposition: that for any given $|C|>1$, one can find a set $(m, n, b)$ such that there are CNGs. Presumably this also extends to CTGs. Actually this proposition is easy to prove in the small NUT limit. There is an unstable circular orbit at $r \simeq 0$, of energy $E_{0} \simeq l^{2} e^{2} / n^{4}$ for $l \neq 0$. From the above, there is a family of corresponding closed null geodesics for

$$
|C| \simeq\left(1+\frac{n^{2}}{4 e^{2}}\right)^{1 / 2} \Longrightarrow|C| \simeq 1+n^{2} / 8 e^{2}
$$

which proves the proposition.

\section{B. Closed charged worldlines}

Since we deal with a charged solution, it is natural to also consider the worldlines of charged particles. In this case we have

$$
\Delta t_{r} \geq \frac{2 \pi}{J f}(E+\kappa \Phi)\left(r^{2}+n^{2}\right)
$$

which is positive as in the geodesic case by virtue of (5.13). The angular contribution $\Delta t_{\theta}$ still satisfies Eq. (6.3) (restricting to $|C| \leq 1$ ) and can be negative. Consider circular orbits at the wormhole throat $r=0$ in the case $f(r)=1, q=0, p=\sqrt{2} n$. From (5.13), $\Delta t_{r}=2 \pi n \gamma / J$, with $\gamma=\sqrt{l^{2}+n^{2}}$, so that the total $\Delta t$ is equal to

$$
\Delta t=\frac{2 \pi n}{J}(\gamma+4 n E-2 J)
$$

with $J=\sqrt{4 n^{2} E^{2}+\gamma^{2}-n^{2}}$. Denoting $\Delta=(\gamma+4 n E)^{2}-4 J^{2}$ and using $E=V_{+}(0)$ given by (5.17) we obtain

$$
\Delta=5\left(\gamma-\gamma_{-}\right)\left(\gamma-\gamma_{-}+\right), \quad \gamma_{ \pm}=-\frac{2 \sqrt{2} \kappa n}{5}\left(1 \pm \sqrt{1-\frac{5}{2 \kappa^{2}}}\right)
$$

Since $\gamma$ must satisfy $\gamma>1$, we find that $\Delta$ is non-positive for

$$
\kappa<-\frac{9}{4 \sqrt{2}}, \quad \gamma_{-} \leq \gamma \leq \gamma_{+}
$$

Note that the upper bound (6.17) is smaller than $4 \sqrt{2}|\kappa| n / 5$, leading to $l^{2} / n^{2}<32 \kappa^{2} / 25$, which is much lower than the upper bound (5.19) of region II, so that the circular worldlines $r=0$ 
satisfying (6.17) are stable. We expect that nearby bound worldlines in the potential well above $r=0$ will similarly violate causality. However, such worldlines are confined to a narrow region around the wormhole throat, so that these causality violations are actually unobservable, for an observer initially living at a large distance $r_{1}$ from the wormhole.

Now let us argue that all the worldlines which can be followed by such an observer returning to his starting point are causal. The negative angular contribution to the total round trip time lapse $\Delta t_{\text {tot }}$ is given by the contribution (6.3) during one period multiplied by a finite number of periods proportional to

$$
\Delta \lambda_{\text {refl }}=2 \int_{r_{0}}^{r_{1}} \frac{d r}{\left(r^{2}+n^{2}\right)\left[(E-V+(r))\left(E-V_{-}(r)\right)\right]^{1 / 2}} .
$$

This will be easily balanced by the positive radial contribution which is proportional to the distance between the turning point $r_{0}$ and $r_{1}$, so that the worldline will remain causal in the large. The observer travelling in a charged rocket and returning to his starting point will not meet his younger self.

However this reasoning fails if, for a given value of the orbital angular momentum $l$, the traveller's energy is just a bit below the maximum of $V_{+}(r)$. The number of $\lambda$-periods (6.18) diverges logarithmically for $r_{0}=r_{\max }$ so that, for $r_{0}$ close to $r_{\max }$, the charged particle makes many turns around the wormhole throat before returning towards infinity. Then, the angular time lapse could be very large (and negative), and the worldline could possibly self-intersect at multiple radii $r=r_{i}$, if the attractor unstable worldline at $r=r_{\max }$ was closed. But we have shown above that the wordlines at $r=0$ can be closed only if they are stable. In the region II parameter range (5.19), there are unstable circular orbits at $r=r_{ \pm}$. During a period, the total $\Delta t$ is

$$
\Delta t=\frac{2 \pi}{J}\left[\left(r_{ \pm}^{2}+n^{2}\right)\left(E+\kappa \Phi\left(r_{ \pm}\right)\right)+4 n^{2} E-2 n \sqrt{4 n^{2} E^{2}+l^{2}}\right]
$$

where $r_{ \pm}$is given by (5.20), $E+\kappa \Phi\left(r_{ \pm}\right)$by (5.23) and $E$ by (5.24). To see whether the square root term may dominate we calculate the difference of the squared quantities $\left[\left(r_{ \pm}^{2}+n^{2}\right)\left(E+\kappa \Phi\left(r_{ \pm}\right)\right)+4 n^{2} E\right]^{2}-4 n^{2}\left(4 n^{2} E^{2}+l^{2}\right)=\frac{8 \kappa^{2} n^{4} l^{2}}{\left(l^{4}-8 \kappa^{2} n^{4}\right)^{2}}\left[l^{6}+2\left(l^{2}+4 n^{2}\right)\left(l^{4}-8 \kappa^{2} n^{4}\right)\right]$

which is positive by virtue of Eq. (5.19), so that these unstable circular wordlines are causal. So, while this does not constitute a rigorous proof, we believe that there are no closed charged wordlines extending to spacelike infinity. The case of wormhole parameters more generic than those (5.15) considered here should similarly be investigated. 


\section{PHYSICAL ASPECTS}

\section{A. Tidal accelerations and traversability}

In order to be traversable, macroscopic Lorentzian wormholes should not only be geodesically complete, with a class of timelike or null geodesics connecting the two asymptotic regions, but they should also be such that the tidal gravitational forces exerted on an observer travelling through remain reasonably small [6]. We will discuss here only the case of radial geodesics $(l=0)$. In the non-relativistic case $(f(r)=1)$, the radial velocity is constant, so the longitudinal tidal force will vanish. However we have noted previously that an extended object falling radially through the wormhole will rotate at a uniform proper angular velocity

$$
\omega(r)=\frac{2 n E}{r^{2}+n^{2}} .
$$

which should generate a centrifugal acceleration $a(\vec{r})$. In flat space, this would be given in cylindrical coordinates $(\rho, z)$ by

$$
a_{\text {flat }}=\ddot{\rho}=\omega^{2}(z) \rho \simeq \frac{4 n^{2}}{\left(r^{2}+n^{2}\right)^{2}} \rho,
$$

where we have assumed for simplicity that the direction of motion is along the $z$ axis, approximated $r \simeq z$ (the radius of the object should be small before the wormhole throat radius $n$ ), and $E \simeq 1$ (non-relativistic limit). Taking as in [6] the reference values $\rho_{\max }=2 \mathrm{~m}$ for the radius of the object and $a_{\max }=g=9.8 \mathrm{~ms}^{-2}$ (Earth gravity), we arrive at the rough estimate at the throat $r=0$

$$
\frac{\ddot{\rho}}{\rho}(0) \simeq \frac{4}{n^{2}} \sim \frac{a_{\max }}{\rho_{\max }} \sim 5 \mathrm{~s}^{-2},
$$

leading to a NUT charge $n \sim 1 \mathrm{~s}$, corresponding to a wormhole throat radius $n \sim 3.10^{8} \mathrm{~m}$ (or in mass units, $\left.n \sim 10^{5} M_{\odot}\right)$.

To improve on this flat-space estimate, we must compute the tidal acceleration between two neighboring points of the freely falling test body with purely spatial separation $\delta x$ in the comoving frame [6], i.e. such that

$$
u_{\mu} \delta x^{\mu}=0
$$

This tidal acceleration is given by the geodesic deviation equation [25]

$$
\delta \ddot{x}^{\mu} \equiv \frac{D^{2}}{d \tau^{2}} \delta x^{\mu}=-R_{\rho \nu \sigma}^{\mu} u^{\rho} \delta x^{\nu} u^{\sigma} .
$$

Before proceeding, we note that in the non-relativistic case the length scale is set by the sole NUT parameter $n$, so that the Riemann tensor components at the throat, and hence the relative tidal accelerations, must be of order $n^{-2}$, as in our flat-spacet estimate. 
In the case of radial geodesics the four-velocity $u^{\mu}=\dot{x}^{\mu}$ has only two non-vanishing components $u^{0}$ and $u^{1}$, so that the condition (7.4) reduces to

$$
\left(g_{00} \delta t+g_{03} \delta \varphi\right) u^{0}+g_{11} \delta r u^{1}=0
$$

which may be used to eliminate $\delta t$ from the right-hand side of (7.5). Taking into account the non-vanishing values of the Riemann tensor components listed in Appendix A, we thus obtain for the longitudinal and transverse accelerations

$$
\begin{aligned}
\delta \ddot{r} & =-\left[R_{t t r}^{r} \delta t u^{0} u^{1}+R_{t r t}^{r} \delta r\left(u^{1}\right)^{2}+R_{t \varphi r}^{r} \delta \varphi u^{0} u^{1}\right] \\
& =\frac{(f-1)\left(3 r^{2}-n^{2}\right)+4 m r}{\left(r^{2}+n^{2}\right)^{2}} \delta r, \\
\delta \ddot{\theta} & =-\left[R^{\theta}{ }_{t \theta t}\left(u^{0}\right)^{2}+R^{\theta}{ }_{r \theta r}\left(u^{1}\right)^{2}\right] \delta \theta \\
& =\frac{r(r-m)-\left(r^{2}-n^{2}\right) f}{\left(r^{2}+n^{2}\right)^{2}} \delta \theta,
\end{aligned}
$$

and a similar equation for $\delta \ddot{\varphi}$. In the non-relativistic case $f=1$ (implying $m=0$ ), the longitudinal acceleration (7.7) vanishes identically, while the transverse acceleration takes the value at the wormhole throat $r=0$

$$
\delta \ddot{\theta}(0)=\frac{1}{n^{2}} \delta \theta
$$

This is precisely one fourth of the flat space estimate (7.3), meaning that our traversability estimate for the NUT charge should be halved, $n \sim 0.5 \mathrm{~s} \sim 1.5 \times 10^{8} \mathrm{~m}$. At present we have no simple explanation for this factor $1 / 4$.

For generic wormhole parameters, the longitudinal and transverse accelerations at the wormhole throat take the values

$$
\begin{aligned}
& \delta \ddot{r}(0)=-\frac{e^{2}-2 n^{2}}{n^{4}} \delta r, \\
& \delta \ddot{\theta}(0)=\frac{e^{2}-n^{2}}{n^{4}} \delta \theta .
\end{aligned}
$$

The transverse (centrifugal) acceleration is always positive $\left(e^{2}-n^{2}>m^{2}\right)$, while the longitudinal acceleration can have either sign. In the small-NUT limit, the two accelerations become large (of order $e^{2} / n^{4}$ ) and opposite in sign, so that they work together to compress the test body along the direction of infall.

\section{B. Electromagnetic fields}

In the present case, the Lorentzian wormhole geometry is generated by electric and/or magnetic fields which will become large near the wormhole throat. For simplicity we discuss only 
the case of purely electric or purely magnetic monopole fields. In Gaussian units these fields are given by

$$
\begin{aligned}
& E=F_{01}=q \frac{r^{2}-n^{2}}{\left(r^{2}+n^{2}\right)^{2}} \quad(p=0), \\
& B=\frac{1}{\sqrt{|g|}} F_{23}=p \frac{r^{2}-n^{2}}{\left(r^{2}+n^{2}\right)^{2}} \quad(q=0),
\end{aligned}
$$

with $e^{2}=q^{2}+p^{2}>n^{2}+m^{2}$ for NUT wormholes. We first consider a purely electric field, in which case $q>n$, so that the maximal field is $E_{\max }>n^{-1}$. A first constraint to be satisfied for the validity of the classical approximation which we have used is that the Schwinger pair creation should be unobservable. In flat space, the Schwinger critical field in $\hbar=c=1$ units reads $E_{\mathrm{Sch}}=\mu_{e}^{2} / e_{0}$, where $\mu_{e}, e_{0}$ are the mass and the charge of the electron. The ratio can be put into the following form

$$
\frac{E_{\text {max }}}{E_{\mathrm{Sch}}}=e_{0} \frac{M_{\odot}}{n} \frac{m_{P l}}{M_{\odot}}\left(\frac{m_{P l}}{\mu_{e}}\right)^{2},
$$

where $m_{P l}$ is the Planck mass. Inserting here $m_{P l} / M_{\odot}=1.1 \times 10^{-38}, m_{P l} / \mu_{e}=2.4 \times 10^{22}, e_{0}=$ $137^{-1 / 2}$ one obtains

$$
\frac{E_{\mathrm{max}}}{E_{\mathrm{Sch}}}=0.54 \times 10^{6} \frac{M_{\odot}}{n} .
$$

Thus in the purely electric case the classical picture is valid if $n \gtrsim 5 \times 10^{5} M_{\odot}$ (which is of the order of the geometric radius of the Sun $\left.R_{\odot} \sim 7 \times 10^{8} \mathrm{~m}\right)$. This lower bound for $n$, which should be improved by considering pair creation in the wormhole spacetime metric is only six times larger than our traversability estimate.

We should also consider the effect of large electric fields on infalling test bodies. While ordinary matter is electrically neutral, so that its motion is not affected by electromagnetic fields, it is made up of charged atomic nuclei and electrons, which are sensitive to these fields. For a spaceship to go through the wormhole without damage, the electric field at the throat should be smaller than the ionization threshold. One can take as the relevant electric field the Coulomb field of the electron at the Bohr radius

$$
E_{i}=\frac{e_{0}}{r_{B}^{2}}, \quad r_{B}=\frac{1}{\mu_{e} e_{0}^{2}}
$$

One finds in the same units:

$$
\frac{E_{i}}{E_{\mathrm{Sch}}}=e_{0}^{6}=137^{-3}=0.4 \times 10^{-6}
$$

Therefore the threshold for traversability of an electric wormhole by an unmanned spaceship is $n \gtrsim 10^{12} M_{\odot} \sim 10^{15} \mathrm{~m} \sim 0.1$ lt-yr. Traversability by a manned spaceship would impose even more drastic constraints. For instance, molecular air is ionized by a constant electric field 
$E \sim 3 \times 10^{6} \mathrm{Vm}^{-1} \sim 10^{-6} E_{i}$; to avoid this the throat radius $n$ should be at least equal to $10^{5}$

lt-yr, of the order of the radius of our Galaxy, which seems totally unrealistic.

In the case of a purely magnetic wormhole no pair creation occurs, but above the Schwinger threshold one can expect phenomena of superstrong magnetic field such as in certain neutron stars. For the wormhole radius value $n \sim 1.5 \times 10^{8} \mathrm{~m}$ derived in the previous subsection, the wormhole-throat field values would be $B(0) \sim 3.3 \times 10^{10} \mathrm{~T}$. For comparison, human exposure to a constant magnetic field of up to $B \sim 8 \mathrm{~T}$ can be tolerated [26]. Taking the latter (magnetic) value as reference would lead to the lower limit for the wormhole radius $n>6 \times 10^{17} \mathrm{~m} \sim 60$ ltyr, corresponding to a huge NUT charge, larger than our previous tidal force estimate by nine orders of magnitude. However, traversability of a purely magnetic NUT wormhole by an unmanned spaceship would seem to be feasible for not too large wormhole radii.

\section{OUTLOOK}

In this paper we have suggested a novel type of wormholes without exotic matter. They are supported by matter sources satisfying the NEC condition and evade the NEC-violation theorem due to the presence of a non-diagonal $(t, \varphi)$ component of the stress tensor, associated with a NUT charge. The price to pay for this is that the spacetime is not strictly asymptotically flat, but only locally asymptotically flat, and presents a Misner string singularity. The particular realization described here is supported by a Maxwell field and corresponds to a supercritically charged RN-NUT solution without a central singularity or horizons. Extending the results of [15], we have analyzed in detail the geodesic motion and shown that the Misner string is transparent for geodesic motion without periodic identification of time, so that the spacetime is geodesically complete. This result is independent of the actual form of the gravitational potential $f(r)$, and holds also for the black-hole and extreme black-hole RN-NUT solutions. A curious effect of the NUT charge is that small freely falling test bodies are endowed with a proper angular momentum or spin, which is aligned with the direction of infall, and is independent of that of the Misner string.

Even without periodic identification of time, the RN-NUT spacetimes contain regions with closed time-like curves. We have shown, however, that for some subfamily of these spacetimes there are no closed timelike or null geodesics, so that freely falling observers should not encounter causality violations. On the other hand, the analysis of the motion of charged test particles has revealed that there are wordlines which can be closed or become past directed if their charge-to-mass ratio is large enough. This was found, however, for orbits threading 
the throat and not reaching asymptotic regions of space-time, so the existence of observable manifestations of non-causality still remains open.

We have also investigated under what conditions these RN-NUT wormholes are macroscopically traversable, and shown that the gravitational tidal forces at the throat could be kept reasonably small for a reasonably small NUT charge. However, the electromagnetic forces acting on ordinary matter under the same conditions would be extremely large, and could be kept under control only in the case of huge NUT charges.

This work could be extended in several directions. First, our analysis was purely classical. One should explore propagation of waves and quantum implications of NUT wormholes, as well as their stability [27]. Also, similar NUT wormhole solutions could presumably be constructed in other gravitating field theories. One such solution was found recently in the Einstein-Skyrme theory [28] after our paper was completed. Five-dimensional vacuum gravity is known to admit NUTless Lorentzian wormhole solutions [29], which are however not traversable [30]. We suggest that the possible existence of geodesically complete and traversable NUTty wormhole solutions to this theory be investigated. Other five-dimensional theories, such as five-dimensional minimal supergravity (which can be consistently truncated to the four-dimensional Einstein-Maxwell theory [31]), should also presumably admit such solutions.

Moreover, by superposing NUT-anti-NUT configurations one could try to construct asymptotically flat wormhole spacetimes. In the extreme black-hole case $\left(b^{2}=0\right)$, one can construct stationary Israel-Wilson-Perjès NUT-anti-NUT superpositions. These are singular if time is periodically identified [32], but can be regular (with only a Misner string connecting the two event horizons) if it is not. From the present analysis, they should be geodesically complete. It would be interesting to extend this to the construction of slowly orbiting NUT-anti-NUT wormhole dynamical solutions in the near-extreme case, i.e. for small $b^{2}>0$. These should be asymptotically flat, with only a non-contractible Misner string loop threading the two wormhole throats. If the parameters could be arranged so that this two-wormhole configuration with two regions at spacelike infinity presented a high degree of symmetry, we speculate that it might be possible to identify these two asymptotically flat regions [33-35] to yield a traversable Wheeler-Misner wormhole [36] connecting two distant parts of a spacetime with only one region at infinity.

We conclude with some astrophysical speculations. Recently it was suggested that wormholes could exist in the galactic centers $[37,38]$ or in the outer regions of halos $[39,40]$. The option to have a wormhole without exotic matter seems attractive: its mass and NUT charge could be reasonably of the scale of the mass concentration in the galactic centers $\left(10^{8}-10^{9} M_{\odot}\right)$, in 
which case it would not be destroyed by quantum effects. However, crucial for this wormhole is the supercritical electric or magnetic charge, whose origin in astrophysical conditions is unclear for the moment. Nevertheless we think that further study of new realizations of gravitational fields with NUTs may be useful for future astrophysical applications.

\section{Acknowledgments}

DG and MG would like to thank LAPTh Annecy-le-Vieux for hospitality at different stages of this work. DG also acknowledges the support of the Russian Foundation of Fundamental Research under the project 14-02-01092-a. MG acknowledges the support of the Ministry of Higher Education and Scientific Research of Algeria (MESRS) under grant 00920090096.

\section{Appendix A: Symmetries}

The metric (2.1) has the following four Killing vectors $K_{(a)}, a=x, y, z, t$ :

$$
\begin{aligned}
& K_{(x)}=-\frac{2 n(1+C \cos \theta) \cos \varphi}{\sin \theta} \partial_{t}-\sin \varphi \partial_{\theta}-\cos \varphi \cot \theta \partial_{\varphi}, \\
& K_{(y)}=-\frac{2 n(1+C \cos \theta) \sin \varphi}{\sin \theta} \partial_{t}+\cos \varphi \partial_{\theta}-\sin \varphi \cot \theta \partial_{\varphi}, \\
& K_{(z)}=\partial_{\varphi}+2 n C \partial_{t}, \\
& K_{(t)}=\partial_{t},
\end{aligned}
$$

the first three forming the algebra $s o(3)$. These formulas extend previously known ones [10-12] to arbitrary $C$-parameter. Note that the presence of $\partial_{t}$-terms in the $s o(3)$ subalgebra reflects the necessity of a compensating time-shift while performing spatial rotations. The algebra so(3) can not be integrated to the group $S O(3)$, but leads to the unitary representation of the group $S U(2)$, provided periodic identification of time according to Misner is performed. This is similar to the case of the magnetic monopole [41], for further references see [11].

The vector potential one-form $A=\Phi(d t-2 n(\cos \theta+C) d \varphi)$ is also symmetric under the action of these four isometries, the Lie derivatives are zero for all $a$ :

$$
\mathcal{L}_{K_{(a)}} A=0=K_{(a)}^{\nu} A_{\mu ; \nu}+K_{(a) ; \mu}^{\nu} A_{\nu}
$$

These symmetries generate four conserved quantities

$$
I_{a}=K_{(a)}^{\mu}\left(\dot{x}^{\nu} g_{\mu \nu}+\kappa A_{\mu}\right)
$$


which read explicitly:

$$
\begin{aligned}
& I_{(x)}=2 n E(\sin \theta)^{-1} \cos \varphi-\sin \theta \dot{\theta}^{2} \rho^{2}-\cot \theta \cos \varphi\left(\rho^{2} \sin ^{2} \theta \dot{\varphi}+2 n E \cos \theta\right), \\
& I_{(y)}=2 n E(\sin \theta)^{-1} \sin \varphi+\cos \theta \dot{\theta}^{2} \rho^{2}-\cot \theta \sin \varphi\left(\rho^{2} \sin ^{2} \theta \dot{\varphi}+2 n E \cos \theta\right), \\
& I_{(z)}=\rho^{2} \sin ^{2} \theta \dot{\varphi}+2 n E \cos \theta, \\
& I_{(t)}=E .
\end{aligned}
$$

They do not depend on the vector potential.

\section{Appendix B: Riemann tensor}

Here we present the Newman-Penrose invariant projections of the Ricci and Weyl tensor for the metric (2.1). Choosing the null tetrad as

$$
\begin{aligned}
l^{\mu} & =\frac{1}{\sqrt{2}}\left(\frac{1}{\sqrt{f}}, \sqrt{f}, 0,0\right), \\
n^{\mu} & =\frac{1}{\sqrt{2}}\left(\frac{1}{\sqrt{f}},-\sqrt{f}, 0,0\right), \\
m^{\mu} & =\left(\frac{\sqrt{2} n \cot \theta}{\sqrt{r^{2}+n^{2}}}, 0, \frac{i}{\sqrt{2} \sqrt{r^{2}+n^{2}}}, \frac{1}{\sqrt{2} \sqrt{r^{2}+n^{2}}}\right), \\
\bar{m}^{\mu} & =\left(\frac{\sqrt{2} n \cot \theta}{\sqrt{r^{2}+n^{2}}}, 0, \frac{-i}{\sqrt{2} \sqrt{r^{2}+n^{2}}}, \frac{1}{\sqrt{2} \sqrt{r^{2}+n^{2}}}\right)
\end{aligned}
$$

we have one non-zero projection of the Ricci tensor

$$
\Phi_{11}=\frac{1}{4} R_{\mu \nu}\left(l^{\mu} n^{\nu}+m^{\mu} \bar{m}^{\nu}\right)=\frac{1}{2} \frac{e^{2}}{\left(r^{2}+n^{2}\right)^{2}},
$$

and one projection of the Weyl tensor:

$$
\begin{aligned}
\Psi_{2} & =C_{\mu \nu \lambda \tau} l^{\mu} m^{\nu} \bar{m}^{\lambda} n^{\tau}=\frac{\left(m^{2}+b^{2}\right)\left(r^{2}-n^{2}\right)+n^{2} r(3 m-2 r)}{\left(r^{2}+n^{2}\right)^{3}} \\
& +i \frac{2 n r\left(m^{2}+b^{2}\right)\left(r^{2}+n^{2}\right)+n r^{4}(r-3 m)+n^{5}(r-m)}{\left(r^{2}+n^{2}\right)^{4}} .
\end{aligned}
$$

These do not depend on the polar angles. The components of the Riemann tensor can be read off easily. We give explicitly the ones used in Sec. 7:

$$
\begin{aligned}
R_{t t r}^{r} & =-f \frac{(f-1)\left(3 r^{2}-n^{2}\right)+4 m r}{\left(r^{2}+n^{2}\right)^{2}}, \\
R_{t r \varphi}^{r} & =R_{\varphi r t}^{r}=-2 n(\cos \theta+C) f \frac{(f-1)\left(3 r^{2}-n^{2}\right)+4 m r}{\left(r^{2}+n^{2}\right)^{2}}, \\
R_{t t \theta}^{\theta} & =R_{t t \varphi}^{\varphi}=-f \frac{r(r-m)-\left(r^{2}-n^{2}\right) f}{\left(r^{2}+n^{2}\right)^{2}}, \\
R_{r r \theta}^{\theta} & =R_{r r \varphi}^{\varphi}=\frac{r(r-m)-\left(r^{2}-n^{2}\right) f}{f\left(r^{2}+n^{2}\right)^{2}},
\end{aligned}
$$


[1] A.H. Taub, Ann. Math. 53, 472 (1951).

[2] E. Newman, L. Tamburino and T. Unti, J. Math. Phys. 4, 915 (1963).

[3] S. W. Hawking and G. F. R. Ellis, The Large Scale Structure of Space-Time, (Cambridge University Press, 1973).

[4] D.R. Brill, Phys. Rev. 133, B845 (1964).

[5] J.A. Wheeler, Geometrodynamics (Academic, New York, 1962).

[6] M.S. Morris and K.S. Thorne, Am. J. Phys. 56, 395 (1988).

[7] M.S. Morris, K.S. Thorne and U. Yurtsever, Phys. Rev. Lett. 61, 1446 (1988).

[8] M. Visser, Lorentzian Wormholes: From Einstein to Hawking (AIP Press, New York, 1995).

[9] E. Teo, Phys. Rev. D58, 024014 (1998) [arXiv:gr-qc/9803098].

[10] C.W. Misner, Journ. Math. Phys. 4, 924 (1963).

[11] J.S. Dowker, Gen. Rel. Grav. 5, 603 (1974).

[12] M. Mueller and M.J. Perry, Class. Quantum Grav. 3 , 65 (1986).

[13] W.B. Bonnor, Proc. Camb. Phil. Soc. 66, 145 (1969).

[14] A. Sackfield, Proc. Camb. Phil. Soc. 70, 89 (1971);

[15] G. Clément, D. Gal'tsov and M. Guenouche, Phys. Lett. B 750 (2015) 591 [arXiv:1508.07622[hepth]].

[16] L. Herrera, N.O. Santos and J.E.F. Skea, Gen. Rel. Grav. 35, 2057 (2003) [arXiv:gr-qc/0306005].

[17] R.G. Vishwakarma, Pramana 85, 1101 (2015) [arXiv:1409.3758].

[18] E. Guendelman, A. Kaganovich, E. Nissimov and S. Pacheva, Open Nucl. Part. Phys. J. 4, 27 (2011) [arXiv:1108.3735 [hep-th]].

[19] V. Kagramanova, J. Kunz, E. Hackmann and C. Lammerzahl, Phys. Rev. D81, 124044 (2010) [arXiv:1002.4342].

[20] R.L. Zimmerman and B.Y. Shahir, Gen. Rel. Grav. 21, 821 (1989).

[21] K. Bronnikov, Acta Phys. Pol. B4, 251 (1973).

[22] H. Ellis, Journ. Math. Phys. 14, 104 (1973).

[23] S. Grunau and V. Kagramanova, Phys. Rev. D81, 044020 (2010) [arXiv:1009.6117].

[24] K.S. Thorne, "Closed Timelike Curves", in Proceedings of the 13th International Conference on General Relativity and Gravitation, edited by R.J. Gleiser, C.N. Kozameh, and O.M. Moreschi, (Institute of Physics Publishing, Bristol, England, 1993), pp. 295-315.

[25] C.W. Misner, K.S. Thorne and J.A. Wheeler, Gravitation (Freeman, San Francisco, 1973). 
[26] D.W. Chakeres, A. Kangarlu, H. Boudoulas and D.C. Young, Journ. Magn. Reson. Imaging 18, $346(2003)$.

[27] G. Holzegel, Class. Quant. Grav. 23, 3951 (2006) [arXiv:gr-qc/0602045].

[28] E. Ayon-Beato, F. Canfora and J. Zanelli, Phys. Lett. B 752, 201 (2016) [arXiv:1509.02659 [gr-qc]].

[29] A. Chodos and S. L. Detweiler, Gen. Rel. Grav. 14, 879 (1982).

[30] M. Azreg-Ainou and G. Clément, Gen. Rel. Grav. 22, 1119 (1990).

[31] G. Clément and D.V. Gal'tsov, Phys. Rev. D 87, 044055 (2013) [arXiv:1301.5084[hep-th]].

[32] J. B. Hartle and S. W. Hawking, Commun. Math. Phys. 26, 87 (1972).

[33] C.W. Misner, Phys. Rev. 118, 1110 (1960).

[34] R.W. Lindquist, Journ. Math. Phys. 4, 938 (1963).

[35] G. Clément, Phys. Rev. D 51, 6803 (1995) [arXiv:gr-qc/9502033]; Journ. Math. Phys. 38, 5807 (1997) [arXiv:gr-qc/9701060].

[36] C.W. Misner and J.A. Wheeler, Ann. Phys. (N.Y.) 2, 525 (1957).

[37] Z. Li and C. Bambi, Phys. Rev. D 90, 024071 (2014) [arXiv:1405.1883 [gr-qc]].

[38] F. Rahaman, P. Salucci, P. K. F. Kuhfittig, S. Ray and M. Rahaman, Annals Phys. 350, 561 (2014) [arXiv:1501.00490 [physics.gen-ph]].

[39] F. Rahaman, P. K. F. Kuhfittig, S. Ray and N. Islam, Eur. Phys. J. C 74, 2750 (2014) [arXiv:1307.1237 [gr-qc]].

[40] P. K. F. Kuhfittig, Eur. Phys. J. C 74, 2818 (2014) [arXiv:1311.2274 [gr-qc]].

[41] C.A. Hurst, Ann. of Phys. 50, 51 (1968). 